This document is confidential and is proprietary to the American Chemical Society and its authors. Do not copy or disclose without written permission. If you have received this item in error, notify the sender and delete all copies.

\title{
Post-Ugi transformations for the access to Pyrrolobenzodiazepine scaffolds with different degree of unsaturation
}

\begin{tabular}{|r|l|}
\hline Journal: & The Journal of Organic Chemistry \\
\hline Manuscript ID & jo-2019-02995k.R1 \\
\hline Manuscript Type: & Article \\
\hline Author: & n/a \\
\hline Complete List of Authors: & $\begin{array}{l}\text { Pertejo, Pablo; Universidad de Burgos Facultad de Ciencias } \\
\text { Carreira-Barral, Israel; Universidad de Burgos Facultad de Ciencias, } \\
\text { Peña-Calleja, Pablo; Universidad de Burgos Facultad de Ciencias } \\
\text { Quesada, Roberto; Universidad de Burgos, Química } \\
\text { Garcia-Valverde, Maria; Universidad de Burgos, Department of } \\
\text { Chemistry }\end{array}$ \\
\hline
\end{tabular}

\section{SCHOLARONE Manuscripts}




\title{
Post-Ugi transformations for the access to Pyrrolobenzodiazepine scaffolds with different degree of unsaturation
}

Pablo Pertejo, Israel Carreira-Barral, Pablo Peña-Calleja, Roberto Quesada, María GarcíaValverde*

Department of Chemistry, Faculty of Science, University of Burgos, 09001, Burgos, Spain

\begin{abstract}
The synthesis of three novel families of pyrrolo[2,1-c][1,4]benzodiazepine-5-ones is described. The compounds were prepared according to a three-step sequence, involving an Ugi reaction, building of the pyrrolo nucleus and reduction-cyclisation to the corresponding diazepine. Depending on the amine employed in the synthesis of the Ugi adducts, different unsaturation degrees could be obtained in the pyrrolo ring (saturated or with endo or exo unsaturations), a key feature determining their biological activity, as it affected the affinity of the pyrrolobenzoadiazepines towards DNA and thus their cytotoxicity. This synthetic methodology represents a significant improvement with respect to those described in the literature so far, as it uses inexpensive and commercially-available starting materials without needing derivatization or the use of protecting groups.
\end{abstract}


<smiles>[R]c1cc(C(=O)O)c([N+](=O)[O-])cc1[R]</smiles>

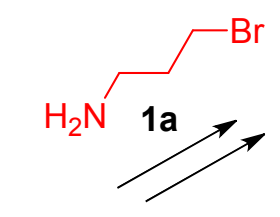<smiles>[R1]NC(=O)C12CCCN1C(=O)c1cc([R])c([R])cc1N=C2C(=C)C=C</smiles>

\section{Introduction}

Pyrrolobenzodiazepines (PBDs) constitute an important class of compounds thoroughly studied due to their activity as antitumour antibiotics. ${ }^{1}$ The cytotoxicity displayed by these compounds is related to their ability to bind covalently to DNA. ${ }^{2}$ Indeed, these compounds have been demonstrated to bind to DNA's minor groove. ${ }^{3} \mathrm{~A}$ key feature for this interaction with DNA, and thus the cytotoxicity of these compounds, is the presence of an imine carbon, or equivalent, at the C11 position. This importance is underscored by the negligible cytotoxicity of analogous compound lacking this imine function. This occurs for instance when the imine group is replaced by an amide, as in PBD dilactames, or by a secondary amine. ${ }^{4}$ Examples of naturally-occurring PBD compounds displaying intriguing pharmacological properties include anthramycin, ${ }^{5}$ chicamycin ${ }^{6}$ and tomaymycin, ${ }^{7}$ all of them featuring an imine carbon, or equivalent, at the C11 position (Figure 1). From the biological perspective it is interesting to remark that the unsaturation degree of the pyrrole core affects the affinity of these systems 
towards DNA as well as their cytotoxicity, both in compounds presenting exo ${ }^{8}$ and $e n d o^{9}$ unsaturations, like tomaymycin and anthramycin, respectively.<smiles>Cc1ccc2c(c1O)N=[13CH][C@H]1CC(/C=C/C(N)=O)=CN1C2=O</smiles>

Anthramycin<smiles>COc1cc2c(cc1O)N=C[C@@H]1C[C@@H](O)CN1C2=O</smiles>

Chicamycin<smiles>C/C=C1/C[C@H]2C=Nc3cc(O)c(OC)cc3C(=O)N2C1</smiles>

Tomaymycin

Figure 1. Examples of naturally-occurring bioactive pyrrolobenzodiazepines.

Numerous methodologies have been developed to synthesize these compounds, ${ }^{10}$ reflecting their interest. The commonest route starts with L-proline, which allows the introduction of the five-membered core. This route was used for the synthesis of DC-81, an antitumor antibiotic produced by Streptomyces species, ${ }^{11}$ following two different strategies: (1) the synthesis of the PBD dilactame as intermediate, followed by the reduction of the carboxylic group derived from proline, ${ }^{12}$ or (2) the formation of the PBD system in the last step, after the proline's carboxylic acid group had been reduced (Figure 2). ${ }^{13}$

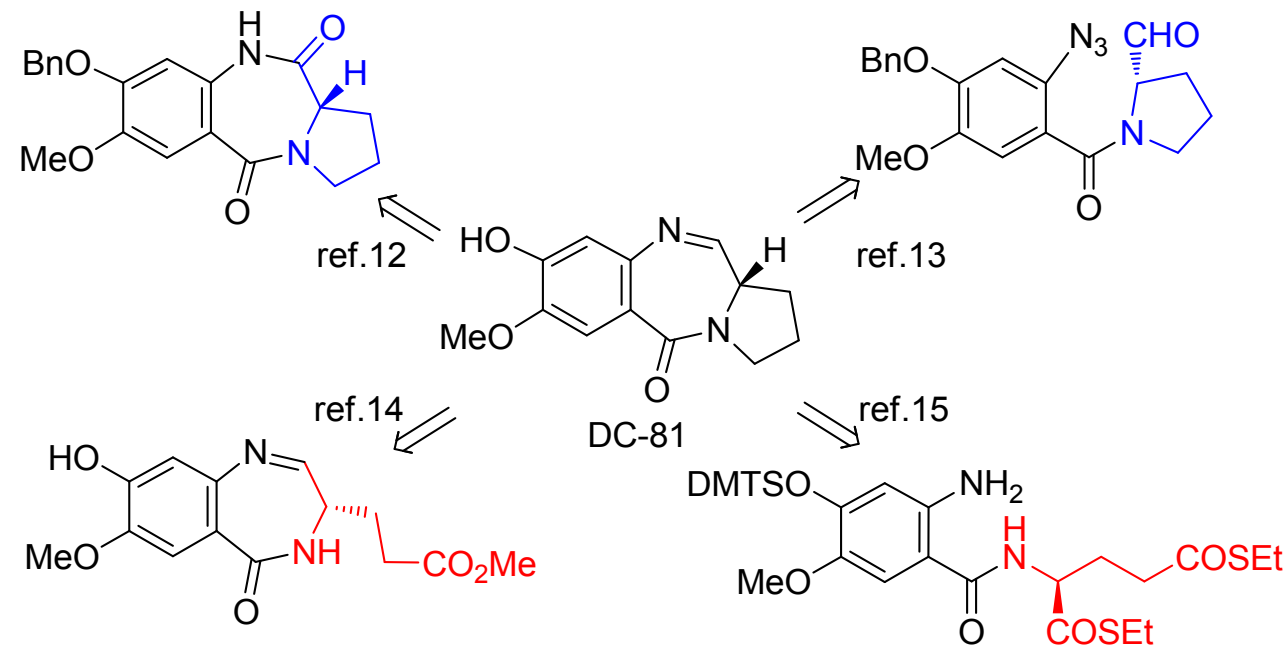

Figure 2. Intermediates in the synthesis of DC-81 starting from L-proline (blue fragment) or L-glutamic acid (red fragment) 
Nevertheless, few results are found in the literature where the precursor of the pyrrole system is an acyclic compound. In these cases, all the fragments which lead to the PBD are linked before the pyrrolo and diazepine cores are obtained, with derivatives of L-glutamic acid as precursors of the pyrrolo system being usually employed. This is the case of the synthetic route starting from L-glutamic acid 5-methyl ester, where the pyrrolo nucleus is obtained in the last step, in a sequence with a low global yield (10\%). ${ }^{14}$ In an effort to improve this yield, a methodology was described employing as a starting material the dithioester derived from Lglutamic acid, an unstable compound. This strategy allowed the simultaneous building of the five- and seven-membered rings in the last step, increasing the global yield up to $33 \%$ (Figure 2). ${ }^{15}$ The third alternative, the construction of the pyrrolo nucleus before the diazepine core, has also been described, but all the methodologies reported so far involve many steps, as well as using specific and usually non-commercial substrates and/or protecting groups, which makes them less appealing than the previous ones. ${ }^{16}$

Prompted by the antitumor activity displayed by these molecules and taking into consideration our previous work with different benzodiazepines, ${ }^{17}$ we envisaged the possibility of synthesizing various pyrrolo[2,1-c][1,4]benzodiazepine-5-ones following analogous methodologies, based on Ugi's reaction. Employing this reaction provides a solution to some of the above-mentioned issues, as it permits the use of commercial reagents which do not need to be derivatized, performing the synthesis under mild conditions and without the use of protecting groups. The compatibility of the isocyanide group with a large number of functional groups allows the introduction of doubly functionalized fragments in the molecule, eventually leading to the desired compounds. Herein, we describe the synthesis of three families of pyrrolo[2,1-c][1,4]benzodiazepine-5-ones derivatives with different unsaturation patterns in the pyrrolo nucleus (Figure 3). The synthetic strategy involves a three-step sequence, namely, an Ugi reaction/pyrrolo synthesis/reduction-cyclization to the diazepines in which the different 
unsaturation degrees on the pyrrolo nucleus is controlled by the nature of the amine and the cyclization methodology employed in the second step.<smiles>[R]NC(=O)C12CCCN1C(=O)c1ccccc1N=C2[Al]</smiles><smiles>[R]NC(=O)C12C=CCN1C(=O)c1ccccc1N=C2[Al]</smiles><smiles>[R]NC(=O)C12C(=O)CCN1C(=O)c1ccccc1N=C2[Te]</smiles>

Figure 3. Pyrrolo[2,1-c][1,4]benzodiazepine-5-ones synthesized in this work ( $R^{1}$ : alkyl, Ar: aryl).

\section{Results and discussion}

The first step in the described synthesis was the Ugi reaction. Three doubly functionalized reagents, two common in all reactions, 2-nitrobenzoic acid derivatives $\mathbf{2}$ and arylglyoxals $\mathbf{3}$, and a different functionalized amine 1 depending on the desired unsaturation degree, namely, 3bromopropylamine (used as its bromide salt) $\mathbf{1 a}$, propargylamine $\mathbf{1 b}$ or $\mathbf{1}$-amino-3-butyne $\mathbf{1 c}$, were reacted with the isocyanide $\mathbf{4}$, allowing the assembly of all the atoms which became part of the final systems in a single step (Scheme 1, Table 1).<smiles>[Z]CN[14CH2]C</smiles>

1a: $\mathrm{Z}=\mathrm{CH}_{2} \mathrm{CH}_{2} \mathrm{Br}$ 1b: $\mathrm{Z}=\mathrm{C} \equiv \mathrm{CH}$ 1c: $\mathrm{Z}=\mathrm{CH}_{2} \mathrm{C}=\mathrm{CH}$<smiles>[R]c1cc(C(=O)O)c([N+](=O)[O-])cc1[R]</smiles>

2a-c

2a: $R^{1}=H, R^{2}: H$

2b: $R^{1}=H, R^{2}: C l$

2c: $\mathrm{R}^{1}=\mathrm{Cl}, \mathrm{R}^{2}: \mathrm{H}$<smiles>[R]c1ccc(C(=O)C=O)cc1</smiles>

3a-d

3a: $\mathrm{R}^{3}=\mathrm{H}$

3b: $\mathrm{R}^{3}=\mathrm{CH}_{3}$

3c: $\mathrm{R}^{3}=\mathrm{Cl}$

3d: $\mathrm{R}^{3}=\mathrm{OCH}_{3}$

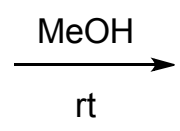

$\stackrel{\ominus}{\mathrm{C}} \equiv \stackrel{\oplus}{\mathrm{N}}-\mathrm{R}^{4}$

$4 a-c$

4a: $\mathrm{R}^{4}=c \mathrm{C}_{6} \mathrm{H}_{11}$

4b: $R^{4}=t B u$

4c: $\mathrm{R}^{4}=n \mathrm{Bu}$<smiles>[Z]CN(C(=O)c1cc([R])c([R])cc1[N+](=O)[O-])/C(C(=O)N[R1])=C(/O)c1ccc([R3])cc1</smiles>

$5 a-i$

Scheme 1. Linking all the fragments through the Ugi reaction. 
Table 1. Results for the Ugi reaction.

\begin{tabular}{|c|c|c|c|c|c|}
\hline Entry & $1(Z)$ & $2\left(R^{1}, R^{2}\right)$ & $3\left(R^{3}\right)$ & $4\left(R^{4}\right)$ & $5^{\mathrm{a}}(\%)$ \\
\hline 1 & 1a $\left(\mathrm{CH}_{2} \mathrm{CH}_{2} \mathrm{Br}\right)$ & $2 a(H, H)$ & 3b $\left(\mathrm{CH}_{3}\right)$ & $4 a\left(c C_{6} \mathrm{H}_{11}\right)$ & $5 \mathbf{b}(70)$ \\
\hline 2 & 1a $\left(\mathrm{CH}_{2} \mathrm{CH}_{2} \mathrm{Br}\right)$ & $2 b(\mathrm{H}, \mathrm{Cl})$ & 3a $(H)$ & $4 \mathbf{a}\left(c \mathrm{C}_{6} \mathrm{H}_{11}\right)$ & $5 f(68)$ \\
\hline 3 & 1b (CECH) & $2 \mathrm{a}(\mathrm{H}, \mathrm{H})$ & $3 a(H)$ & $4 a\left(c C_{6} \mathrm{H}_{11}\right)$ & $5 j(69)$ \\
\hline 4 & $\mathbf{1 b}(\mathrm{C} \equiv \mathrm{CH})$ & $2 a(H, H)$ & $3 b\left(\mathrm{CH}_{3}\right)$ & $4 \mathbf{a}\left(c \mathrm{C}_{6} \mathrm{H}_{11}\right)$ & $\mathbf{5 k}(79)$ \\
\hline 5 & 1b (CECH) & $2 \mathbf{a}(H, H)$ & 3d $\left(\mathrm{OCH}_{3}\right)$ & $4 \mathbf{a}\left(c \mathrm{C}_{6} \mathrm{H}_{11}\right)$ & $5 I(47)$ \\
\hline 6 & $\mathbf{1 b}(\mathrm{C} \equiv \mathrm{CH})$ & $2 a(H, H)$ & 3a $(H)$ & $\mathbf{4 b}(t \mathrm{Bu})$ & $5 \mathrm{~m}(53)$ \\
\hline 7 & $\mathbf{1 b}(\mathrm{C} \equiv \mathrm{CH})$ & $2 b(\mathrm{H}, \mathrm{Cl})$ & 3a $(H)$ & 4a $\left(c \mathrm{C}_{6} \mathrm{H}_{11}\right)$ & 5n (72) \\
\hline 8 & 1b $(\mathrm{C} \equiv \mathrm{CH})$ & $2 \mathrm{c}(\mathrm{Cl}, \mathrm{H})$ & 3d $(H)$ & $4 a\left(c C_{6} \mathrm{H}_{11}\right)$ & $50(70)$ \\
\hline 9 & 1c $\left(\mathrm{CH}_{2} \mathrm{C} \equiv \mathrm{CH}\right)$ & $2 a(H, H)$ & 3d $(H)$ & $4 a\left(c C_{6} \mathrm{H}_{11}\right)$ & $5 p(87)$ \\
\hline 10 & 1c $\left(\mathrm{CH}_{2} \mathrm{C} \equiv \mathrm{CH}\right)$ & $2 a(H, H)$ & 3d $(H)$ & $\mathbf{4 b}(t \mathrm{Bu})$ & $5 q(82)$ \\
\hline 11 & 1c $\left(\mathrm{CH}_{2} \mathrm{C} \equiv \mathrm{CH}\right)$ & $2 \mathrm{c}(\mathrm{Cl}, \mathrm{H})$ & 3d $(H)$ & $4 a\left(c C_{6} \mathrm{H}_{11}\right)$ & $5 r(82)$ \\
\hline
\end{tabular}

a 5 a $5 c-5 e, 5 g-5 i$ Ugi adduct intermediates for the synthesis of $6 a, 6 c-6 e, 6 g-i$ were not isolated but used directly in the next step

The results were similar regardless of the starting amine and the substitution of the other components. Interestingly, no spontaneous cyclization was observed upon the formation of Ugi adducts 5a-i, derived from 3-bromopropylamine $\mathbf{1 a}$ as it was described for the 3bromopropionic acid derivatives. ${ }^{17 b}$

The second step in these syntheses was the construction of the pyrrolo core from the Ugi adducts, which underscores the importance of the functionalization in the Ugi reactants to carry out the post-condensation reactions towards the desired intermediates.

In order to obtain the pyrrolidines $\mathbf{6}$, Ugi adducts $\mathbf{5 b}$ and $\mathbf{5} \mathbf{f}$ were isolated, in $\mathbf{7 0}$ and $80 \%$ yield respectively, (Table 1, entries 1 and 2 ) and treated with cesium carbonate (1.5 equiv.), yielding the corresponding pyrrolidine almost quantitatively (93 and $94 \%$ yield of isolated pure pyrrolidines $\mathbf{6 b}$ and $\mathbf{6 f}$ respectively). However, the overall yield was improved when the Ugi/cyclization was carried out in a single step, i. e., adding the cesium carbonate to the reaction mixture in the Ugi reaction (Table 2, entries 2 and 6). This is probably due to the loss of the Ugi adduct during the purification process. In this way, pyrrolidines $6 a-i$ were 
synthesized in a single step from the four reactants through an Ugi reaction, followed by a base-promoted intramolecular nucleophilic substitution (Scheme 2).

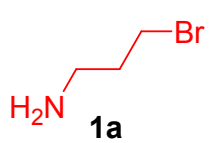<smiles>[R]c1cc(C(=O)O)c([N+](=O)[O-])cc1[R]</smiles>

2a-c

2a: $R^{1}=H, R^{2}: H$

2b: $R^{1}=H, R^{2}: C l$ 2c: $\mathrm{R}^{1}=\mathrm{Cl}, \mathrm{R}^{2}: \mathrm{H}$<smiles>[R]c1ccc(C(=O)C=O)cc1</smiles>

3a: $\mathrm{R}^{3}=\mathrm{H}$

3b: $\mathrm{R}^{3}=\mathrm{CH}_{3}$

3c: $\mathrm{R}^{3}=\mathrm{Cl}$

3d: $\mathrm{R}^{3}=\mathrm{OCH}_{3}$

$\stackrel{\ominus}{\mathrm{C}} \stackrel{\oplus}{\mathrm{N}}-\mathrm{R}^{4}$

4a-c

4a: $\mathrm{R}^{4}=c_{6} \mathrm{H}_{11}$

4b: $R^{4}=t B u$

4c: $\mathrm{R}^{4}=n \mathrm{Bu}$<smiles>[R]NC(=O)C1(C(=O)c2ccc([R])cc2)CCCN1C(=O)c1cc([R])c([R])cc1[N+](=O)[O-]</smiles>

Scheme 2. Synthesis of pyrrolidines 6a-i from 3-bromopropylamine.

Table 2. Results for the synthesis of pyrrolidines 6a-i in a single step.

\begin{tabular}{|c|c|c|c|c|}
\hline Entry & $2\left(R^{1}, R^{2}\right)$ & $3\left(R^{3}\right)$ & $4\left(R^{4}\right)$ & $6(\%)$ \\
\hline 1 & $2 a(H, H)$ & $3 a(H)$ & $4 a\left(c C_{6} \mathrm{H}_{11}\right)$ & $6 a(86)$ \\
\hline 2 & $2 a(H, H)$ & $\mathbf{3 b}\left(\mathrm{CH}_{3}\right)$ & $4 \mathrm{a}\left(c \mathrm{C}_{6} \mathrm{H}_{11}\right)$ & $6 b(89)^{a}$ \\
\hline 3 & $2 a(H, H)$ & $3 c(\mathrm{Cl})$ & $4 \mathrm{a}\left(c \mathrm{C}_{6} \mathrm{H}_{11}\right)$ & $6 c(81)$ \\
\hline 4 & $2 a(H, H)$ & $3 a(H)$ & $\mathbf{4 b}(t \mathrm{Bu})$ & 6d (80) \\
\hline 5 & $2 a(H, H)$ & $3 a(H)$ & 4c $(n \mathrm{Bu})$ & $6 e(87)$ \\
\hline 6 & $2 b(\mathrm{H}, \mathrm{Cl})$ & $3 a(H)$ & $4 a\left(c C_{6} \mathrm{H}_{11}\right)$ & $6 f(84)^{a}$ \\
\hline 7 & $2 \mathrm{c}(\mathrm{Cl}, \mathrm{H})$ & $\mathbf{3 b}\left(\mathrm{CH}_{3}\right)$ & $4 a\left(c C_{6} \mathrm{H}_{11}\right)$ & 6g (87) \\
\hline 8 & $2 \mathrm{c}(\mathrm{Cl}, \mathrm{H})$ & 3a $(H)$ & $\mathbf{4} \mathbf{b}(t \mathrm{Bu})$ & 6h (76) \\
\hline 9 & $2 \mathrm{c}(\mathrm{Cl}, \mathrm{H})$ & 3d $\left(\mathrm{OCH}_{3}\right)$ & $4 a\left(c C_{6} \mathrm{H}_{11}\right)$ & $6 i(86)$ \\
\hline
\end{tabular}

a The two-steps overall yield was $65 \%$ for $\mathbf{6 b}$ and $64 \%$ for $\mathbf{6 f}$.

The synthesis of dihydropyrroles was carried out from the Ugi adducts derived from propargylamine $\mathbf{1 b}$. Two different strategies were examined as shown in Scheme $\mathbf{3}$ and Table 3: (a) addition of metal salts, which would favor the formation of a complex between the dicarbonyl system, the alkyne and the metal ion; coordination would make the alkyne an 
electrophile and would increase the nucleophilicity of the enol (Scheme 4), ${ }^{18}$ and (b) addition of bases, which would favor the formation of the allene from the propargyl anion, hence making the terminal carbon an electrophile due to a hyperconjugative effect, as well as an enolate (Scheme 5). ${ }^{19}$
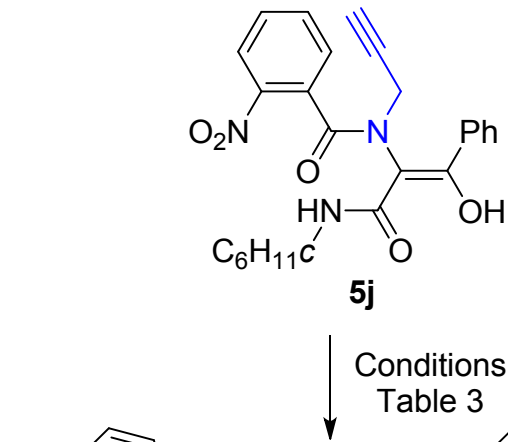<smiles>CCCCCCCCCCCCCC1(C(=O)c2ccccc2)C=CCN1C(=O)c1ccccc1[N+](=O)[O-]</smiles>

$7 a$<smiles>C=CCCCCCCCCCCCCCC(=O)C1(C(=O)c2ccccc2)C(=C)CN1C(=O)c1ccccc1[N+](=O)[O-]</smiles>

9<smiles>CCCCCCCCCCCCCC1(C(=O)c2ccccc2)CC=CN1C(=O)c1ccccc1[N+](=O)[O-]</smiles>

8<smiles>CCCCCCCCCC(=O)N1CC=CC1C(=O)c1ccccc1[N+](=O)[O-]</smiles>

10

Scheme 3. Cyclization of Ugi adduct 5j derived from 3-propargylamine. 
Table 3. Conditions for the cyclization of Ugi adduct 5j derived from 3-propargylamine.

\begin{tabular}{cccccccccc}
\hline \multirow{2}{*}{ Entry } & \multirow{2}{*}{ Reagent } & \multirow{2}{*}{ Equiv. } & \multirow{2}{*}{ Solvent } & \multirow{2}{*}{$(\mathbf{h})$} & \multirow{2}{*}{$\left({ }^{\circ} \mathbf{C}\right)$} & \multicolumn{4}{c}{ Products $^{\mathrm{a}}$} \\
\cline { 7 - 10 } & & & & & & $\mathbf{7 a}$ & $\mathbf{8}$ & $\mathbf{9}$ & $\mathbf{1 0}$ \\
\hline $\mathbf{2 n C l}$ & 0.05 & Chloroform & 24 & 60 & 74 & - & 26 & - \\
$\mathbf{2}$ & $\mathrm{AgClO}_{4}$ & 0.05 & Chloroform & 24 & 60 & 87 & - & - & 13 \\
$\mathbf{3}$ & $\mathrm{AgClO}_{4}$ & 0.05 & Toluene & 24 & 110 & 100 & - & - & - \\
$\mathbf{4}$ & $t \mathrm{BuOK}$ & 2.5 & $\mathrm{THF}$ & 1 & 20 & 34 & - & - & 66 \\
$\mathbf{5}$ & $t \mathrm{BuOK}$ & 1 & THF & 1 & 20 & 66 & - & - & 34 \\
$\mathbf{6}$ & $\mathrm{Na}_{2} \mathrm{CO}_{3}$ & 2 & Acetonitrile & 6 & 80 & 100 & - & - & - \\
\hline
\end{tabular}

${ }^{\text {a }}$ Ratio obtained by ${ }^{1} \mathrm{H}$ NMR spectroscopy.

Firstly, different reaction conditions were tried in the presence of $\operatorname{In}(I I I)$, a well-known Lewis acid, ${ }^{20}$ as its chloride salt. Although 3 -pyrroline 7 a was systematically obtained, it was not the only product. Unexpectedly, methyleneazetidinone $\mathbf{9}$ was also formed (Table 3, entry 1). The mechanism that drives the formation of both compounds would be similar: $\ln ($ III) would coordinate simultaneously to the 1,3-dicarbonyl system and the alkyne ( $\sigma$ and $\pi$-coordination, respectively) and then the enolate would attack the triple bond intramolecularly (Scheme 4). However, the formation of $\mathbf{7}$ is favored over that of $\mathbf{9}$, since the former takes place through a 5-endo-dig attack and the latter through a 4-exo-dig one although, according to Baldwin's rules, ${ }^{21}$ the 4-exo-dig attack is not favored. 


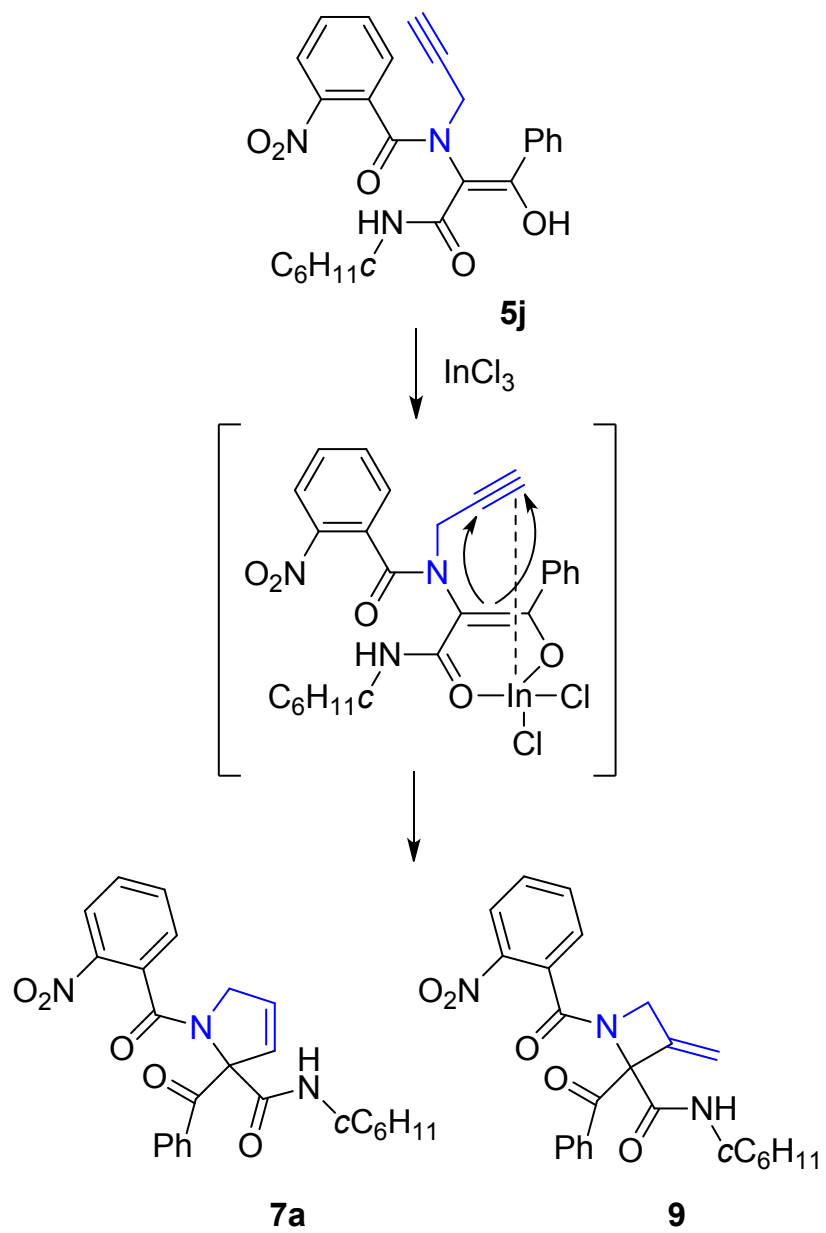

Scheme 4. Cyclization mechanism of Ugi adducts derived from 3-propargylamine promoted by $\operatorname{lnCl}{ }_{3}$

Interestingly, when the reaction was conducted in boiling chloroform in the presence of silver(I) perchlorate instead of indium(III) chloride, only the 5-endo-dig cyclization occurred; however, the debenzoylated product $\mathbf{1 0}$ was obtained together with the desired 3-pyrroline $\mathbf{7 a}$ (Table 3, entry 2). In order to avoid the formation of the former, the reaction was performed in boiling toluene, a solvent with lower water content than chloroform; in this way, 3-pyrroline 7a was obtained exclusively (Table $\mathbf{3}$, entry 3 ).

To address the cyclization reaction from a second perspective, i. e., the addition of bases, potassium tert-butoxide and sodium carbonate were tried. The former is a common reagent for the formation of allenes from propargyl systems so, initially, 2.5 equiv. of 
potassium tert-butoxide were added to a solution of $5 \mathbf{j}$ in THF and the reaction was carried out at room temperature. Although 2,3-dihydropyrrole 8 would be the expected product, ${ }^{22}$ a mixture of $7 a$ and 10 was obtained, the latter being the major one (Table 3, entry 4). In view of this, the amount of base was reduced to one equivalent while keeping the remaining conditions (Table 3, entry 5), resulting in that, although 7 a became the major product, the debenzoylated one $\mathbf{1 0}$ was still formed due to the nucleophilicity of the base.

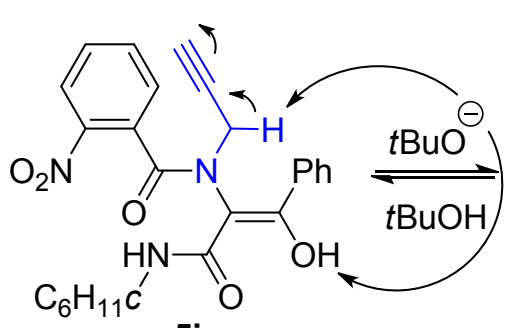

$5 \mathbf{j}$
$\mathrm{C}_{6} \mathrm{H}_{11} \mathrm{C}$

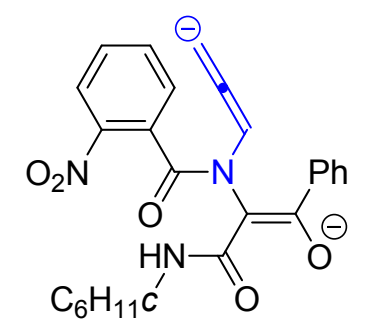

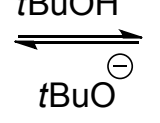<smiles>C1CCCC1</smiles>

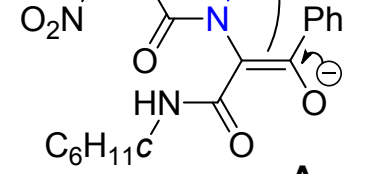

A

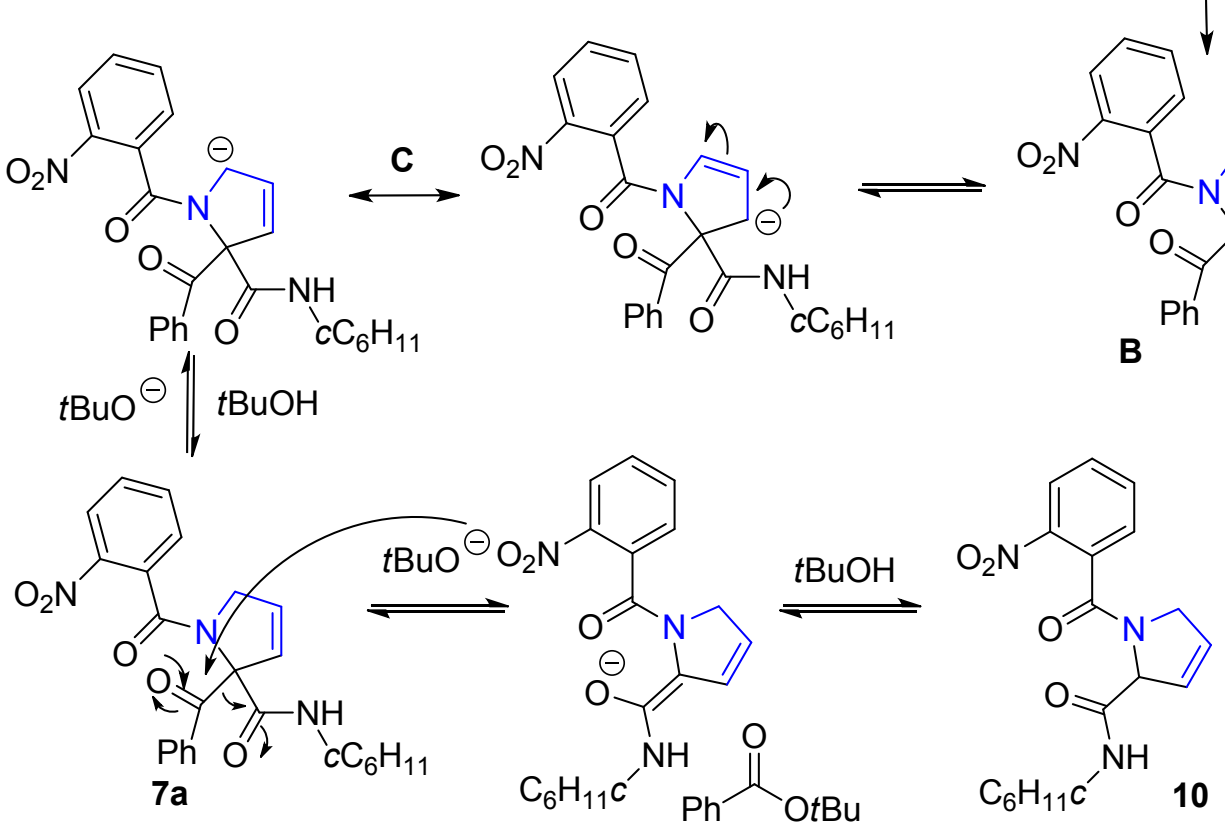

Scheme 5. Proposed mechanism for the formation of 3-pyrrolines $\mathbf{7 a}$ and $\mathbf{1 0}$ promoted by bases

In an attempt to explain the formation of the 3-pyrroline 7a instead of the expected 2pyrroline $8,^{22}$ Vazquez and colleagues have proposed a mechanism for an analogous system according to which the enolate would attack the alkyne's terminal carbon. ${ }^{23}$ However, the 
base-induced isomerization of propargyl amides to allenamides is well documented ${ }^{24}$ and, moreover, it is favoured in our reaction conditions (Table 3, entries 4 and 5). ${ }^{25} \ln$ this way, a plausible mechanism would start with the isomerization to the allenamide (A), which would undergo an intramolecular attack on the terminal carbon from the enolate (5-endo-trig cyclization), thus giving rise to vinyl carbanion B. The translocation of the allyl proton would originate an allyl anion (C), thus accounting for the isomerization of the double bond. ${ }^{26}$ The protonation of $\mathbf{C}$ would lead to 3-pyrroline 7 a since they are more stable than 2 -pyrrolines. ${ }^{27}$ The retro-Claisen reaction on this 3-pyrroline 7 a would yield the debenzoylated 3-pyrroline $\mathbf{1 0}$ (Scheme 5).

With the aim of avoiding the debenzoylation reaction, the less nucleophilic sodium carbonate was employed as the base. Sodium carbonate is also less basic, so the temperature was increased. Typically, this kind of bases requires the use of boiling $\mathrm{DMF}^{28}$ or of THF combined with a microwave oven, ${ }^{29}$ but surprisingly the reaction leading to 3-pyrroline $7 a$ was quantitative in boiling acetonitrile (Table 3, entry 6). This approach is also safer and more ecofriendly than the use of silver(I) perchlorate. Therefore, a series of 3-pyrrolines was synthesized according to Scheme 6 with good yields (Table 4).<smiles>[R]NC(=O)/C(=C(/O)c1ccc([R])cc1)N(CC#C)C(=O)c1cc([R2])c([R1])cc1[N+](=O)[O-]</smiles>

$5 \mathrm{j}-\mathrm{O}$
$\mathrm{AcN}, \Delta$

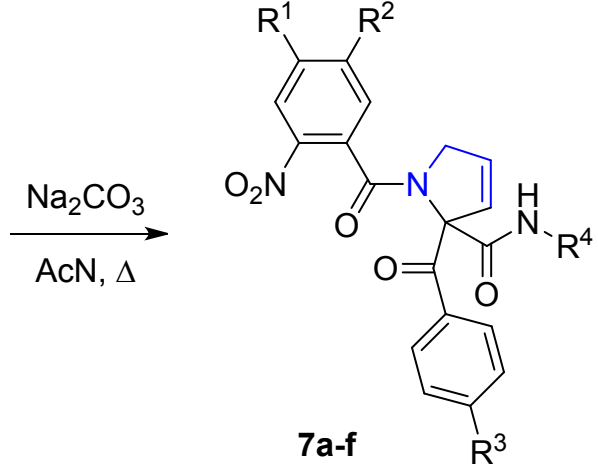

Scheme 6. Synthesis of 3-pyrrolines 7a-f from Ugi adducts 5j-0. 
Table 4. Global yields for the synthesis of 3-pyrrolines 7a-f from Ugi adducts 5j-0.

\begin{tabular}{|c|c|c|}
\hline Entry & $5\left(R^{1}, R^{2}, R^{3}, R^{4}\right)$ & 7 (\%) \\
\hline 1 & $5 \mathbf{j}\left(\mathrm{H}, \mathrm{H}, \mathrm{H}, c \mathrm{C}_{6} \mathrm{H}_{11}\right)$ & $7 a(81)$ \\
\hline 2 & $5 \mathbf{k}\left(\mathrm{H}, \mathrm{H}, \mathrm{CH}_{3}, c \mathrm{C}_{6} \mathrm{H}_{11}\right)$ & $7 b(73)$ \\
\hline 3 & $5 \mathrm{I}\left(\mathrm{H}, \mathrm{H}, \mathrm{OCH}_{3}, \mathrm{CC}_{6} \mathrm{H}_{11}\right)$ & $7 c(82)$ \\
\hline 4 & $5 \mathrm{~m}(\mathrm{H}, \mathrm{H}, \mathrm{H}, t \mathrm{Bu})$ & $7 d(72)$ \\
\hline 5 & 5n $\left(\mathrm{H}, \mathrm{Cl}, \mathrm{H}, c \mathrm{C}_{6} \mathrm{H}_{11}\right)$ & 7e (77) \\
\hline 6 & $50\left(\mathrm{Cl}, \mathrm{H}, \mathrm{H}, \mathrm{cC}_{6} \mathrm{H}_{11}\right)$ & $7 f(87)$ \\
\hline
\end{tabular}

Finally, the syntheses of the pyrrolidines incorporating an exo unsaturation in the pyrrolo core were conducted from Ugi adducts $5 p$-r derived from 1-amino-3-butyne 1c. Although the Ugi adducts were initially subjected to a basic treatment, employing both sodium carbonate and sodium tert-butoxide, in order to obtain the desired 3-methylenepyrrolidines, these trials were unsuccessful and the starting materials were recovered. The formation of the allene in these conditions is not favored, as the hydrogen atoms of the methylene group adjacent to the triple bond are not as acidic as are those of the propargylic position. This also confirmed that the attack of the enolate to the triple bond, which would eventually lead to the formation of the pyrrolic ring, is not favored. In light of these results, a study similar to that described in the previous section was performed. Hence, Ugi adduct $\mathbf{5 p}$ was dissolved in boiling toluene and indium(III) chloride or silver(I) perchlorate (0.05 equiv.) were added to the solution. These conditions favored the 5-exo-dig cyclization reaction, driving to the regioselective and exclusive formation of 3-methylenepyrrolidine 11a. Thus, we chose the safer indium(III) chloride as catalyst affording thereby the corresponding 3methylenepyrrolidine in good yields (Scheme 7, Table 5). 


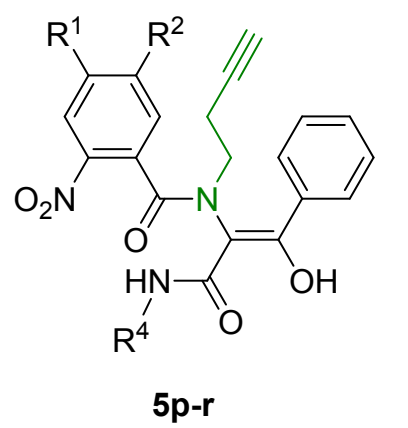<smiles>[R1]NC(=O)C1(C(=O)c2ccccc2)C(=C)CCN1C(=O)c1cc([R])c([R1])cc1[N+](=O)[O-]</smiles>

Scheme 7. Synthesis of 3-methylenepyrrolidines 11a-c from Ugi adducts 5p-r.

Table 5. Results for the synthesis of 3-methylenepyrrolidines 11a-c from Ugi adducts 5p-r.

\begin{tabular}{ccc}
\hline Entry & $\mathbf{5}\left(\mathrm{R}^{1}, \mathrm{R}^{2}, \mathrm{R}^{4}\right)$ & $\mathbf{1 1}(\%)$ \\
\hline 1 & $5 \mathrm{p}\left(\mathrm{H}, \mathrm{H}, c \mathrm{C}_{6} \mathrm{H}_{11}\right)$ & $11 \mathrm{a}(88)$ \\
2 & $5 \mathbf{q}(\mathrm{H}, \mathrm{H}, t \mathrm{Bu})$ & $11 \mathbf{b}(78)$ \\
3 & $5 \mathrm{r}\left(\mathrm{Cl}, \mathrm{H}, c \mathrm{C}_{6} \mathrm{H}_{11}\right)$ & $11 \mathrm{c}(70)$ \\
\hline
\end{tabular}

The final cyclisation step furnishing the diazepine ring was carried out in a similar way for all the different pyrrolo systems, 6, 7 and 11. Reduction of the nitro group with tin(II) chloride in the presence of $\mathrm{HCl}$ in hot ethanol lead to a spontaneous intramolecular cyclization. ${ }^{17}$ The generated amino group undergo a nucleophilic addition to the carbonyl group of the molecule giving rise to an imine fragment. This process generated compounds 12a-r in good to moderate yields (Scheme 8). 


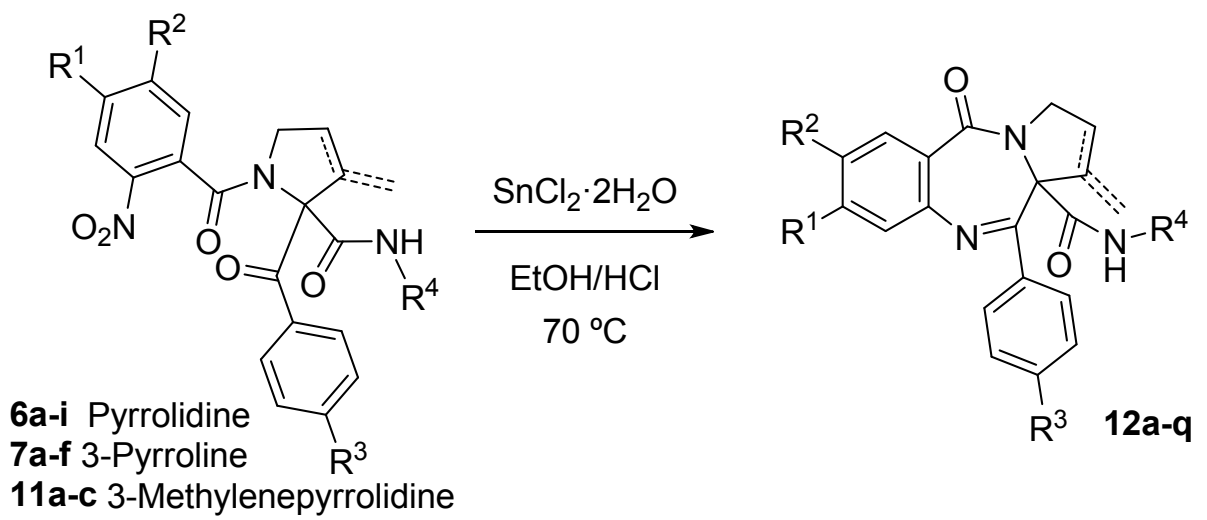<smiles>O=C(NC1CCCCC1)C1(CCCN2CCCCC2)C(=O)c2ccccc2N=C1c1ccccc1</smiles><smiles>CCCCC1(C(=O)NC2CCCCC2)C(=O)c2ccccc2N=C1c1ccc(C)cc1</smiles><smiles></smiles><smiles>CC(C)(C)NC(=O)C12CCCN1C(=O)c1ccccc1N=C2c1ccccc1</smiles><smiles>CCCCNC(=O)C12CCCN1C(=O)c1ccccc1N=C2c1ccccc1</smiles><smiles>CCCCC1(C(=O)NC2CCCCC2)C(=O)c2cc(I)ccc2N=C1c1ccccc1</smiles>

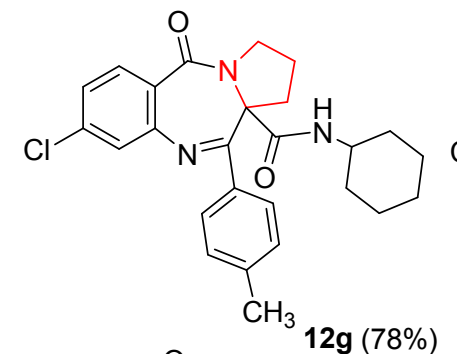<smiles>CC(C)(C)NC(=O)C12CCCN1C(=O)c1ccc(Cl)cc1N=C2c1ccccc1</smiles>

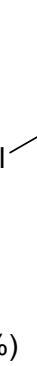<smiles></smiles><smiles>O=C1c2ccccc2N=C(c2ccccc2)C2(C(=O)NC3CCCCC3)C=CCN1C2=O</smiles><smiles>O=C1c2ccccc2N=C(c2ccc(Cl)cc2)C2(C(=O)NC3CCCCC3)C=CCN12</smiles><smiles></smiles>

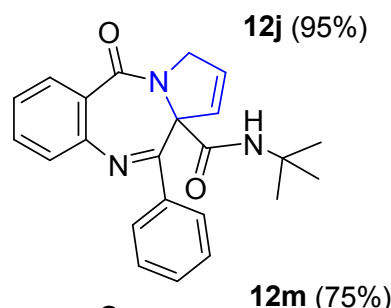<smiles>CCCCCCCCCC1=C(C(=O)NC2CCCCC2)C12CCN1C(=O)c3ccccc3N=C(c3ccccc3)C12</smiles>

$12 p(60 \%)$

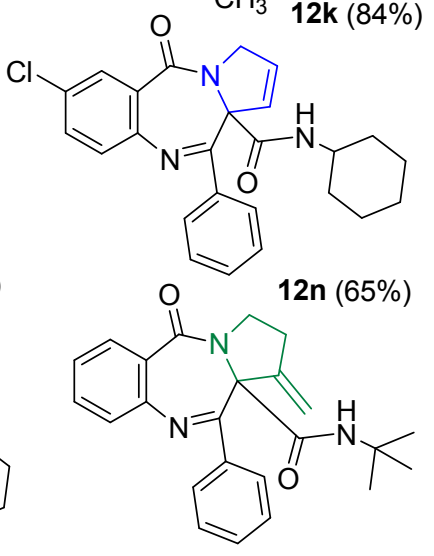<smiles>COC(=O)C1=Nc2cc(Cl)ccc2C(=O)N2CC=CC12C(=O)NC1CCCCC1</smiles>

$12 q(58 \%)$

12r (62\%) 
Scheme 8. Synthesis of pyrrolobenzodiazepine-5-ones 12a-r from different pyrrolo systems.

X-ray diffraction studies of compound $\mathbf{1 2} \mathbf{i}$ showed that the conformation adopted by these systems is determined by the configuration of the stereogenic centre C3 (3S-(M)- and 3R-(P)-conformers), as the largest substituent, the amide group derived from the isocyanide component in the Ugi reaction, prefers the pseudoaxial orientation (Figure 4). ${ }^{30}$

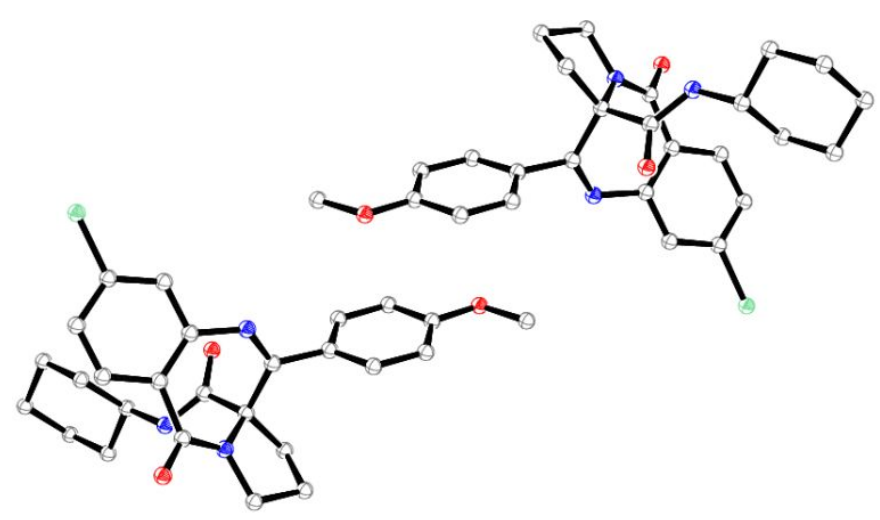

Figure 4. Crystal packing in the X-ray structure of compound 12i. Hydrogen atoms and two chloroform molecules have been omitted for the sake of simplicity. The ORTEP plot is at the $30 \%$ probability level. The molecule on the left side of the image is the $3 \mathrm{R}-(P)$ - conformer, while that on the right side is the 3S-(M)- conformer.

\section{Conclusion}

In this work we have described the use of Ugi/post-condensation methodologies to provide straightforward access to three novel families of pyrrolobenzodiazepine-5-ones. Selecting the appropriate amine, it is possible to obtain saturated and unsaturated pyrrolic cores bearing endo or exo unsaturations in just three steps. This methodology represents a remarkable improvement with respect to those starting from acyclic compounds previously reported, as the starting materials are affordable and commercially available, and there is no need for derivatization, neither to use protecting groups. This allows the syntheses of three different 
pyrrolobenzodiazepine-5-one families with high atom economy and high to moderate global yields. 


\section{Experimental section}

\section{General methods}

Melting points have not been corrected. ${ }^{1} \mathrm{H}$ and ${ }^{13} \mathrm{C}$ NMR spectra were recorded in $\mathrm{CDCl}_{3}$ at 300 and $75 \mathrm{MHz}$, respectively, on a Varian Mercury 300 system; DEPT-135 experiments were conducted to assign carbon-13 signals. Chemical shifts are reported in parts per million with respect to residual solvent protons and coupling constants in hertz. High resolution mass spectra were recorded in positive ion mode by electronic impact (El; Agilent 7010 mass spectrometer with a triple quadrupole analizer) at $70 \mathrm{eV}$ or electrospray ionization (ESI; Agilent 6545 with a quadrupole-time-of-flight analyzer).

General procedure for the synthesis of Ugi adducts $5 b, f, j-r$. The corresponding amine $\mathbf{1 a}-\mathbf{c}$ (1.0 mmol, 1.0 equiv.) (3-bromopropylamine 1a should be obtained from the treatment of the commercial 3-bromopropylamine hydrobromide ( $1.1 \mathrm{mmol}, 1.1$ equiv.) with sodium hydroxide (1.0 mmol, 1.0 equiv.) in methanol) and the corresponding arylglyoxal 3a-d (1.0 mmol, 1.0 equiv., $0.1 \mathrm{M})$ which were dissolved in methanol $(10 \mathrm{~mL})$ and the resulting solution was stirred at room temperature for 15 minutes to preform the imine. Subsequently, the corresponding 2nitrobenzoic acid 2a-c (1.0 mmol, 1.0 equiv.) and isocyanide 4a-c (1.0 mmol, 1.0 equiv.) derivatives were added, and the mixture was stirred at room temperature for 24 hours. The precipitate formed, corresponding to the different Ugi adducts $\mathbf{5}$, was isolated by vacuum filtration and dried. Note: as two rotamers are observed in the NMR spectra of these compounds, the terms "major" and "minor" are used to indicate to which of the rotamers the corresponding signal is assigned.

(E)-N-(3-Bromopropyl)-N-(3-(cyclohexylamino)-1-hydroxy-3-oxo-1-(p-tolyl)prop-1-en-2-yl)-2nitrobenzamide (5b). White solid. Yield: $381 \mathrm{mg}, 70 \%$. M. p. $96-97{ }^{\circ} \mathrm{C}$ (as 85:15 rotamer mixture). ${ }^{1} \mathrm{H} N M R\left(300 \mathrm{MHz}, \mathrm{CDCl}_{3}\right) \delta$ (major rotamer): $15.32(\mathrm{~s}, 1 \mathrm{H}, \mathrm{OH}), 8.22(\mathrm{~d}, J=8.6 \mathrm{~Hz}$, 1H), 7.79-7.02 (m, 7H), $6.67(\mathrm{~d}, J=8.4 \mathrm{~Hz}, 1 \mathrm{H}, \mathrm{NH}), 3.95-3.79(\mathrm{~m}, 1 \mathrm{H}), 3.61-3.34(\mathrm{~m}, 1 \mathrm{H}), 3.23-$ 
$3.11(\mathrm{~m}, 1 \mathrm{H}), 3.05-2.88(\mathrm{~m}, 2 \mathrm{H}), 2.43(\mathrm{~s}, 3 \mathrm{H}), 2.07-1.15(\mathrm{~m}, 12 \mathrm{H}) .{ }^{13} \mathrm{C}$ NMR $\{$ DEPT-135\} $(75 \mathrm{MHz}$, $\left.\mathrm{CDCl}_{3}\right) \delta$ (major rotamer): $171.5(\mathrm{Cq}), 169.2(\mathrm{Cq}), 141.0(\mathrm{Cq}), 135.0(\mathrm{CH}), 132.9(\mathrm{Cq}), 131.7(\mathrm{Cq})$, $129.9(\mathrm{CH}), 129.2(\mathrm{CH}), 128.2(\mathrm{CH}), 127.5(\mathrm{CH}), 127.4(\mathrm{CH}), 125.3(\mathrm{CH}), 125.0(\mathrm{Cq}), 104.7(\mathrm{Cq})$, $50.7\left(\mathrm{CH}_{2}\right), 49.0(\mathrm{CH}), 32.9\left(\mathrm{CH}_{2}\right), 32.6\left(\mathrm{CH}_{2}\right), 30.1\left(\mathrm{CH}_{2}\right), 25.4\left(\mathrm{CH}_{2}\right), 25.2\left(\mathrm{CH}_{2}\right), 25.1\left(\mathrm{CH}_{2}\right), 21.6$ $\left(\mathrm{CH}_{3}\right)$. HRMS (ESI-QTOF) $\mathrm{m} / \mathrm{z}$ : calculated for $\mathrm{C}_{26} \mathrm{H}_{31} \mathrm{BrN}_{3} \mathrm{O}_{5}\left[\mathrm{M}+\mathrm{H}^{+}\right]$544.1442; found 544.1443.

(E)-N-(3-Bromopropyl)-5-chloro-N-(3-(cyclohexylamino)-1-hydroxy-3-oxo-1-phenylprop-1-en2-yl)-2-nitrobenzamide (5f). White solid. Yield: $384 \mathrm{mg}, 68 \%$. M. p. $145-146{ }^{\circ} \mathrm{C}$ (as 88:12 rotamer mixture). ${ }^{1} \mathrm{H} \mathrm{NMR}\left(300 \mathrm{MHz}, \mathrm{CDCl}_{3}\right) \delta$ (major rotamer): $15.28(\mathrm{~s}, 1 \mathrm{H}, \mathrm{OH}), 8.17(\mathrm{~d}, J=$ 8.9 Hz, 1H), 7.59-7.50 (m, 6H), 7.09 (s, 1H), $6.57(\mathrm{~d}, J=7.7 \mathrm{~Hz}, 1 \mathrm{H}, \mathrm{NH}), 3.92-3.81(\mathrm{~m}, 1 \mathrm{H}), 3.20-$ $2.86(\mathrm{~m}, 4 \mathrm{H}), 2.08-1.10(\mathrm{~m}, 12 \mathrm{H}) .{ }^{13} \mathrm{C}$ NMR $\left\{\right.$ DEPT-135\} $\left(75 \mathrm{MHz}, \mathrm{CDCl}_{3}\right) \delta$ (major rotamer): $171.6(\mathrm{Cq}), 169.0(\mathrm{Cq}), 167.6(\mathrm{Cq}), 142.9(\mathrm{Cq}), 142.0(\mathrm{Cq}), 134.4(\mathrm{Cq}), 130.9(\mathrm{CH}), 130.3(\mathrm{CH})$, 128.6 (CH), $127.6(\mathrm{CH}), 127.4(\mathrm{CH}), 126.7(\mathrm{CH}), 104.8(\mathrm{Cq}), 50.5\left(\mathrm{CH}_{2}\right), 49.0(\mathrm{CH}), 32.9\left(\mathrm{CH}_{2}\right)$, 32.6 $\left(\mathrm{CH}_{2}\right), 32.2\left(\mathrm{CH}_{2}\right), 25.3\left(\mathrm{CH}_{2}\right), 25.2\left(\mathrm{CH}_{2}\right), 25.1\left(\mathrm{CH}_{2}\right)$. HRMS (ESI-QTOF) m/z: calculated for $\mathrm{C}_{25} \mathrm{H}_{28} \mathrm{BrClN}_{3} \mathrm{O}_{5}\left[\mathrm{M}+\mathrm{H}^{+}\right]$564.0895; found 564.0893.

(E)-N-(3-(Cyclohexylamino)-1-hydroxy-3-oxo-1-phenylprop-1-en-2-yl)-2-nitro-N-(prop-2-yn-1yl)benzamide (5j). Light pink solid. Yield: $309 \mathrm{mg}, 69 \%$. M. p. $154-156{ }^{\circ} \mathrm{C}$ (as 55:45 rotamer mixture). ${ }^{1} \mathrm{H} \mathrm{NMR}\left(300 \mathrm{MHz}, \mathrm{CDCl}_{3}\right.$ ) $\delta: 15.77(\mathrm{~s}, 1 \mathrm{H}, \mathrm{OH}$, minor), 15.37 (s, $1 \mathrm{H}, \mathrm{OH}$, major), 8.24$6.46(\mathrm{~m}, 10 \mathrm{H}), 5.06(\mathrm{dd}, J=17.0,2.5 \mathrm{~Hz}, 1 \mathrm{H}$, minor), 4.28-4.05 (m, 2H, minor), 3.95-3.85 (m, $1 \mathrm{H}$, major), 3.86 (dd, $J=17.0,2.5 \mathrm{~Hz}, 1 \mathrm{H}$, minor), 3.74 (dd, $J=17.9,2.5 \mathrm{~Hz}, 1 \mathrm{H}$, major), 3.75-3.65 (m, 1H, minor), 3.55 (dd, $J=17.9,2.5 \mathrm{~Hz}, 1 \mathrm{H}$, major), $2.53(\mathrm{t}, J=2.5 \mathrm{~Hz}, 1 \mathrm{H}$, minor), 2.09 (t, $J=$ $2.5 \mathrm{~Hz}, 1 \mathrm{H}$, major), 2.04-1.01 (m, 10H). ${ }^{13} \mathrm{C} N M R\left\{{ }^{1} \mathrm{H}\right\}\left(75 \mathrm{MHz}, \mathrm{CDCl}_{3}\right)$ 8: 171.7, 170.2, 144.9, $134.8,134.5,131.3,130.5,129.0,128.4,128.3,128.3,127.7,127.5,127.3,125.3,124.6,106.2$ $77.6,76.5,74.9,74.6,49.3,49.0,42.6,41.1,32.7,32.6,32.2,25.4,25.3,25.2$.

(E)-N-(3-(Cyclohexylamino)-1-hydroxy-3-oxo-1-(p-tolyl)prop-1-en-2-yl)-2-nitro-N-(prop-2-yn1-yl)benzamide (5k). White solid. Yield: $364 \mathrm{mg}, 79 \%$. M. p. $178-180{ }^{\circ} \mathrm{C}$ (as 55:45 rotamer 
mixture). ${ }^{1} \mathrm{H}$ NMR (300 MHz, $\mathrm{CDCl}_{3}$ ) $\delta: 15.74(\mathrm{~s}, 1 \mathrm{H}, \mathrm{OH}$, major), 15.38 (s, $1 \mathrm{H}, \mathrm{OH}$, minor), 8.27$6.56(\mathrm{~m}, 9 \mathrm{H}), 5.07$ (dd, $J=17.0,2.5 \mathrm{~Hz}, 1 \mathrm{H}$, major), 4.27-4.08 ( $\mathrm{m}, 2 \mathrm{H}$, minor), 3.83 (dd, $J=17.0$, $2.5 \mathrm{~Hz}, 1 \mathrm{H}$, major), 3.99-3.77 (m, 1H, minor), 3.76 (dd, $J=18.0,2.5 \mathrm{~Hz}, 1 \mathrm{H}$, minor), 3.75-3.60 (m, $1 \mathrm{H}$, major), 3.54 (dd, $J=18.0,2.5 \mathrm{~Hz}, 1 \mathrm{H}$, minor), 2.51 (t, $J=2.5 \mathrm{~Hz}, 1 \mathrm{H}$, major), 2.43 (s, 3H, major), $2.43\left(\mathrm{~s}, 3 \mathrm{H}\right.$, minor), $2.10\left(\mathrm{t}, J=2.5 \mathrm{~Hz}, 1 \mathrm{H}\right.$, minor), 2.03-0.83 (m, 10H). ${ }^{13} \mathrm{C} \mathrm{NMR}\left\{{ }^{1} \mathrm{H}\right\}(75$ $\left.\mathrm{MHz}, \mathrm{CDCl}_{3}\right)$ $\delta: 171.7,170.1,169.4,168.9,145.3,144.5,141.9,140.7,134.9,134.6,133.5$ $133.1,131.6,131.3,131.1,130.6,130.5,130.2,129.7,129.3,129.0,128.5,128.2,127.7,127.4$ $127.3,125.3,124.9,124.6,110.0,107.2,106.0,77.7,76.6,74.8,74.6,49.2,49.0,49.0,42.6$, $40.9,32.7,32.6,32.6,32.3,25.4,25.3,25.2,25.1,25.0,25.0,24.7,21.7,21.6$.

(E)-N-(3-(Cyclohexylamino)-1-hydroxy-1-(4-methoxyphenyl)-3-oxoprop-1-en-2-yl)-2-nitro- $N$ (prop-2-yn-1-yl)benzamide (5I). White solid. Yield: 224 mg, 47\%. M. p. 170-172 ${ }^{\circ} \mathrm{C}$ (as 55:45 rotamer mixture). ${ }^{1} \mathrm{H}$ NMR (300 MHz, $\left.\mathrm{CDCl}_{3}\right) \delta: 15.79$ (s, $1 \mathrm{H}, \mathrm{OH}$, major), 15.40 (s, $1 \mathrm{H}, \mathrm{OH}$, minor), 8.33-6.57 (m, 9H), 5.08 (dd, $J=17.0,2.5 \mathrm{~Hz}, 1 \mathrm{H}$, major), 5.08 (dd, J = 17.0, $2.5 \mathrm{~Hz}, 1 \mathrm{H}$, major), 4.30-4.04 (m, 2H, minor), $3.88(\mathrm{~s}, 3 \mathrm{H}$, minor), $3.86(\mathrm{~s}, 3 \mathrm{H}$, major), 3.78 (dd, $J=17.9,2.5$ $\mathrm{Hz}, 1 \mathrm{H}$, minor), $3.95-3.80(\mathrm{~m}, 1 \mathrm{H}$, minor), $3.75-3.55$ ( $\mathrm{m}, 1 \mathrm{H}$, major), 3.57 (dd, $J=17.9,2.5 \mathrm{~Hz}$, $1 \mathrm{H}$, minor), $2.50\left(\mathrm{t}, J=2.5 \mathrm{~Hz}, 1 \mathrm{H}\right.$, major), $2.09\left(\mathrm{t}, J=2.5 \mathrm{~Hz}, 1 \mathrm{H}\right.$, major), $2.10-0.94(\mathrm{~m}, 10 \mathrm{H}) .{ }^{13} \mathrm{C}$ NMR $\left\{{ }^{1} \mathrm{H}\right\}\left(75 \mathrm{MHz}, \mathrm{CDCl}_{3}\right) \delta: 171.4,169.5,164.0,161.8,161.3,134.6,133.4,130.9,130.6$, $130.5,130.2,129.6,129.2,128.5,128.0,127.4,125.7,125.3,124.9,124.6,114.4,113.9,113.7$ $106.6,77.7,74.7,74.5,55.5,55.5,49.2,49.1,49.0,42.6,40.7,32.7,32.6,32.3,25.4,25.3,25.1$, 25.0, 24.8.

(E)-N-(3-(tert-Butylamino)-1-hydroxy-3-oxo-1-phenylprop-1-en-2-yl)-2-nitro-N-(prop-2-yn-1-

yl)benzamide $(5 \mathrm{~m})$. White solid. Yield: $223 \mathrm{mg}, 53 \%$. M. p. $186-188{ }^{\circ} \mathrm{C}$ (as $55: 45$ rotamer mixture). ${ }^{1} \mathrm{H} \mathrm{NMR}\left(300 \mathrm{MHz}, \mathrm{CDCl}_{3}\right.$ ) $\delta: 15.84$ (s, $1 \mathrm{H}, \mathrm{OH}$, minor), 15.51 (s, $1 \mathrm{H}, \mathrm{OH}$, major), 8.28$6.22(\mathrm{~m}, 10 \mathrm{H}), 5.05(\mathrm{dd}, J=17.1,2.5 \mathrm{~Hz}, 1 \mathrm{H}$, minor), 4.31-4.10 (m, 2H, minor), $3.95(\mathrm{dd}, J=$ 17.1, $2.5 \mathrm{~Hz}, 1 \mathrm{H}$, minor), 3.75 (dd, $J=17.9,2.5 \mathrm{~Hz}, 1 \mathrm{H}$, major), 3.56 (d, $J=17.9,2.5 \mathrm{~Hz}, 1 \mathrm{H}$, major), $2.55(\mathrm{t}, \mathrm{J}=2.5 \mathrm{~Hz}, 1 \mathrm{H}$, minor), $2.13(\mathrm{t}, \mathrm{J}=2.5 \mathrm{~Hz}, 1 \mathrm{H}$, major), 1.52 (s, 9H, minor), 1.42 (s, 
$9 \mathrm{H}$, major). ${ }^{13} \mathrm{C}$ NMR $\left\{{ }^{1} \mathrm{H}\right\}\left(75 \mathrm{MHz}, \mathrm{CDCl}_{3}\right) \delta: 171.9,170.6,170.2,169.8,168.2,144.8,144.4$, $134.8,134.6,133.6,131.1,130.7,130.4,130.3,130.1,128.9,128.6,128.4,128.3,127.7,127.4$ $127.2,125.1,124.9,124.3,107.9,106.6,77.7,76.7,75.0,74.5,52.7,52.4,42.7,41.5,28.8$ $28.4,28.3$.

\section{(E)-5-Chloro-N-(3-(cyclohexylamino)-1-hydroxy-3-oxo-1-phenylprop-1-en-2-yl)-2-nitro- $N$ -}

(prop-2-yn-1-yl)benzamide (5n). Light pink solid. Yield: $347 \mathrm{mg}, 72 \%$. M. p. $144-146{ }^{\circ} \mathrm{C}$ (as 57:43 rotamer mixture). ${ }^{1} \mathrm{H}$ NMR (300 MHz, $\left.\mathrm{CDCl}_{3}\right) \delta: 15.76(\mathrm{~s}, 1 \mathrm{H}, \mathrm{OH}$, major), $15.29(\mathrm{~s}, 1 \mathrm{H}$, $\mathrm{OH}$, minor), 8.19-6.93 (m, 8H), $6.66(\mathrm{~d}, J=7.9 \mathrm{~Hz}, 1 \mathrm{H}$, minor), $6.59(\mathrm{~d}, J=7.7 \mathrm{~Hz}, 1 \mathrm{H}$, major), $5.88(\mathrm{~d}, J=2.4 \mathrm{~Hz}, 1 \mathrm{H}$, major), 5.04 (dd, $J=17.0,2.4 \mathrm{~Hz}, 1 \mathrm{H}$, major), 4.27-4.10 (m, 2H, minor), 4.02 (dd, $J=17.0,2.4 \mathrm{~Hz}, 1 \mathrm{H}$, major), 3.97-3.81 (m, $1 \mathrm{H}$, minor), 3.74 (dd, $J=18.0,2.5 \mathrm{~Hz}, 1 \mathrm{H}$, minor), 3.74 (dd, $J=18.0,2.5 \mathrm{~Hz}, 1 \mathrm{H}$, minor), $3.73-3.65$ (m, $1 \mathrm{H}$, major), 3.57 (dd, $J=18.0,2.5$ $\mathrm{Hz}, 1 \mathrm{H}$, minor), $2.61(\mathrm{t}, J=2.5 \mathrm{~Hz}, 1 \mathrm{H}$, major), $2.13(\mathrm{t}, J=2.5 \mathrm{~Hz}, 1 \mathrm{H}$, minor), 2.08-1.06 (m, 10H). ${ }^{13} \mathrm{C} N M R\left\{{ }^{1} \mathrm{H}\right\}\left(75 \mathrm{MHz}, \mathrm{CDCl}_{3}\right) \delta: 171.8,171.6,169.2,168.7,166.7,166.6,140.5,134.4,133.8$, 133.6, 133.0, 131.2, 130.6, 130.5, 130.2, 129.2, 128.8, 128.6, 128.5, 128.4, 127.6, 127.3, 127.3, $126.6,125.7,110.0,107.2,106.3,75.4,74.8,49.5,49.0,42.7,42.1,32.7,32.2,25.4,25.1,25.0$, 24.9.

\section{(E)-4-Chloro-N-(3-(cyclohexylamino)-1-hydroxy-3-oxo-1-phenylprop-1-en-2-yl)-2-nitro- $N$ -}

(prop-2-yn-1-yl)benzamide (5o). White solid. Yield: 337 mg, 70\%. M. p. 168-170 ${ }^{\circ} \mathrm{C}$ (as 52:48 rotamer mixture). ${ }^{1} \mathrm{H}$ NMR (300 MHz, $\mathrm{CDCl}_{3}$ ) $\delta: 15.80$ (s, $1 \mathrm{H}, \mathrm{OH}$, major), 15.32 (s, $1 \mathrm{H}, \mathrm{OH}$, minor), 8.33-7.11 (m, 7H), $6.93(\mathrm{~d}, J=8.2 \mathrm{~Hz}, 1 \mathrm{H}$, minor), $6.64(\mathrm{~d}, J=8.1 \mathrm{~Hz}, 1 \mathrm{H}), 6.27(\mathrm{~d}, J=8.3$ $\mathrm{Hz}, 1 \mathrm{H}$, major), 5.04 (dd, $J=17.0,2.4 \mathrm{~Hz}, 1 \mathrm{H}$, major), 4.29-4.03 (m, $2 \mathrm{H}$, minor), 3.89 (dd, $J=$ 17.0, $2.4 \mathrm{~Hz}, 1 \mathrm{H}$, major), 3.95-3.85 ( $\mathrm{m}, 1 \mathrm{H}$, minor), 3.80-3.67 ( $\mathrm{m}, 1 \mathrm{H}$, major), 3.72 ( $\mathrm{dd}, J=18.0$, $2.5 \mathrm{~Hz}, 1 \mathrm{H}$, minor), 3.56 (dd, $J=18.0,2.5 \mathrm{~Hz}, 1 \mathrm{H}$, minor), $2.55(\mathrm{t}, J=2.5 \mathrm{~Hz}, 1 \mathrm{H}$, major), 2.10 (t, $J$ $=2.5 \mathrm{~Hz}, 1 \mathrm{H}$, minor), 2.00-0.92 (m, 10H). ${ }^{13} \mathrm{C} N M R\left\{{ }^{1} \mathrm{H}\right\}\left(75 \mathrm{MHz}, \mathrm{CDCl}_{3}\right) \delta: 171.8,171.8,170.5$, $169.2,168.7,145.4,136.6,136.1,134.9,134.4,133.4,131.4,130.5,129.4,129.0,128.6,128.4$, 
$127.7,127.4,125.4,124.7,106.3,77.5,76.4,75.1,74.8,49.4,49.0,42.7,41.4,32.7,32.6,32.3$, $25.4,25.3,25.1,25.0,24.9$.

(E)-N-(But-3-yn-1-yl)-N-(3-(cyclohexylamino)-1-hydroxy-3-oxo-1-phenylprop-1-en-2-yl)-2nitrobenzamide (5p). White solid. Yield: $401 \mathrm{mg}, 87 \%$. M. p. $178-180{ }^{\circ} \mathrm{C}$ (as $84: 16$ rotamer mixture). ${ }^{1} \mathrm{H}$ NMR (300 MHz, $\left.\mathrm{CDCl}_{3}\right) \delta$ (major rotamer): $15.41(\mathrm{~s}, 1 \mathrm{H}, \mathrm{OH}), 8.24-7.08(\mathrm{~m}, 9 \mathrm{H})$, $6.67(\mathrm{~d}, J=7.8 \mathrm{~Hz}, 1 \mathrm{H}, \mathrm{NH}), 3.95-3.78(\mathrm{~m}, 1 \mathrm{H}), 3.23-3.12(\mathrm{~m}, 1 \mathrm{H}), 2.97-2.86(\mathrm{~m}, 1 \mathrm{H}), 2.25-0.70$ (m, 13H). ${ }^{13} \mathrm{C}$ NMR \{DEPT-135\} (75 MHz, $\mathrm{CDCl}_{3}$ ) $\delta$ (major rotamer): $171.6(\mathrm{Cq}), 169.2(\mathrm{Cq}), 168.7$ (Cq), $145.0(\mathrm{Cq}), 134.8\left(\mathrm{CH}_{\mathrm{Ar}}\right), 134.4(\mathrm{Cq}), 132.7(\mathrm{Cq}), 130.7\left(\mathrm{CH}_{\mathrm{Ar}}\right), 130.4\left(\mathrm{CH}_{\mathrm{Ar}}\right), 128.5\left(\mathrm{CH}_{\mathrm{Ar}}\right)$, 127.4 (CH), $127.1(\mathrm{CH}), 125.4(\mathrm{CH}), 104.6(\mathrm{Cq}), 80.0(\mathrm{Cq}), 70.6(\mathrm{CH}), 51.1\left(\mathrm{CH}_{2}\right), 49.1(\mathrm{CH}), 32.8$ $\left(\mathrm{CH}_{2}\right), 32.6\left(\mathrm{CH}_{2}\right), 25.4\left(\mathrm{CH}_{2}\right), 25.2\left(\mathrm{CH}_{2}\right), 25.1\left(\mathrm{CH}_{2}\right), 17.6\left(\mathrm{CH}_{2}\right)$. HRMS (ESI-QTOF) $\mathrm{m} / \mathrm{z}$ : calculated for $\mathrm{C}_{26} \mathrm{H}_{28} \mathrm{~N}_{3} \mathrm{O}_{5}\left[\mathrm{M}+\mathrm{H}^{+}\right]$462.2023; found 462.2027.

(E)-N-(But-3-yn-1-yl)-N-(3-(tert-butylamino)-1-hydroxy-3-oxo-1-phenylprop-1-en-2-yl)-2-

nitrobenzamide (5q). White solid. Yield: $357 \mathrm{mg}, 82 \%$. M. p. $124-126{ }^{\circ} \mathrm{C}$ (as $82: 18$ rotamer mixture). ${ }^{1} \mathrm{H}$ NMR (300 MHz, $\left.\mathrm{CDCl}_{3}\right) \delta$ (major rotamer): $15.59(\mathrm{~s}, 1 \mathrm{H}, \mathrm{OH}), 8.22-7.02(\mathrm{~m}, 9 \mathrm{H})$, $6.55(\mathrm{~s}, 1 \mathrm{H}, \mathrm{NH}), 3.56-1.99(\mathrm{~m}, 4 \mathrm{H}), 1.85(\mathrm{t}, \mathrm{J}=2.6 \mathrm{~Hz}, 1 \mathrm{H}), 1.52(\mathrm{~s}, 9 \mathrm{H}) .{ }^{13} \mathrm{C}$ NMR $\{$ DEPT-135\} $(75$ $\left.\mathrm{MHz}, \mathrm{CDCl}_{3}\right) \delta$ (major rotamer): $171.8(\mathrm{Cq}), 170.2(\mathrm{Cq}), 168.8(\mathrm{Cq}), 144.8(\mathrm{Cq}), 134.6(\mathrm{CH}), 132.6$ (Cq), $130.3(\mathrm{CH}), 129.0(\mathrm{CH}), 128.5(\mathrm{CH}), 127.4(\mathrm{CH}), 127.1(\mathrm{CH}), 125.2(\mathrm{CH}), 104.9(\mathrm{Cq}), 80.1$ (Cq), $70.6(\mathrm{CH}), 52.5(\mathrm{Cq}), 51.2\left(\mathrm{CH}_{2}\right), 28.8\left(\mathrm{CH}_{3}\right), 17.5\left(\mathrm{CH}_{2}\right)$. HRMS (EI) $\mathrm{m} / \mathrm{z}$ : calculated for $\mathrm{C}_{24} \mathrm{H}_{26} \mathrm{~N}_{3} \mathrm{O}_{5}\left[\mathrm{M}+\mathrm{H}^{+}\right]$436.1867; found 436.1874 .

(E)-N-(But-3-yn-1-yl)-4-chloro-N-(3-(cyclohexylamino)-1-hydroxy-3-oxo-1-phenylprop-1-en-2yl)-2-nitrobenzamide (5r). White solid. Yield: $407 \mathrm{mg}, 82 \%$. M. p. $122-124{ }^{\circ} \mathrm{C}$ (as 82:18 rotamer mixture). ${ }^{1} \mathrm{H}$ NMR (300 MHz, $\left.\mathrm{CDCl}_{3}\right) \delta$ (major rotamer): $15.38(\mathrm{~s}, 1 \mathrm{H}, \mathrm{OH}), 8.20-6.97(\mathrm{~m}, 8 \mathrm{H})$, $6.57(\mathrm{~d}, J=8.0 \mathrm{~Hz}, 1 \mathrm{H}, \mathrm{NH}), 3.97-3.72(\mathrm{~m}, 1 \mathrm{H}), 3.20-3.10(\mathrm{~m}, 1 \mathrm{H}), 2.95-2.84(\mathrm{~m}, 1 \mathrm{H}), 2.25-0.81$ (m, 10H). ${ }^{13} \mathrm{C}$ NMR $\{$ DEPT-135\} (75 MHz, CDCl $)$ ) $\delta$ (major rotamer): 171.6 (Cq), $169.0(\mathrm{Cq}), 167.8$ (Cq), $136.3(\mathrm{Cq}), 134.9\left(\mathrm{CH}_{\mathrm{Ar}}\right), 134.3(\mathrm{Cq}), 130.9(\mathrm{Cq}), 130.7\left(\mathrm{CH}_{\mathrm{Ar}}\right), 129.2\left(\mathrm{CH}_{\mathrm{Ar}}\right), 128.6\left(\mathrm{CH}_{\mathrm{Ar}}\right)$, 
$128.3\left(\mathrm{CH}_{\text {Ar }}\right), 127.7\left(\mathrm{CH}_{\text {Ar }}\right), 127.3\left(\mathrm{CH}_{\text {Ar }}\right), 125.5\left(\mathrm{CH}_{\text {Ar }}\right), 104.5(\mathrm{Cq}), 80.0(\mathrm{CH}), 70.8(\mathrm{CH}), 51.1$ $\left(\mathrm{CH}_{2}\right), 48.9(\mathrm{CH}), 32.7\left(\mathrm{CH}_{2}\right), 32.6\left(\mathrm{CH}_{2}\right), 29.6\left(\mathrm{CH}_{2}\right), 25.4\left(\mathrm{CH}_{2}\right), 25.1\left(\mathrm{CH}_{2}\right), 17.5\left(\mathrm{CH}_{2}\right)$. HRMS (EI) $\mathrm{m} / \mathrm{z}$ : calculated for $\mathrm{C}_{26} \mathrm{H}_{27} \mathrm{ClN}_{3} \mathrm{O}_{5}\left[\mathrm{M}+\mathrm{H}^{+}\right]$496.1634; found 496.1641 .

General procedure for the synthesis of pyrrolidines $6 a-i .1 .0 \mathrm{mmol}$ (1.0 equiv.) of sodium hydroxide was added to a solution of 3-bromopropylamine hydrobromide 1a (1.1 mmol, 1.1 equiv.) in methanol $(10 \mathrm{~mL})$. Subsequently, the corresponding arylglyoxal $\mathbf{3 a}-\mathbf{d}$ (1.0 mmol, 1.0 equiv., $0.1 \mathrm{M}$ ) was added, followed by the addition of the corresponding 2-nitrobenzoic acid 2a-c (1.0 mmol, 1.0 equiv.) and the corresponding isocyanide 4a-c (1.0 mmol, 1.0 equiv.). The resulting solution was stirred at room temperature for 24 hours and, then, cesium carbonate (1.5 mmol, 1.5 equiv.) was added. The mixture was stirred at room temperature for one hour and the solvent was removed under reduced pressure. The raw product was dissolved in dichloromethane and washed with acidified water. The organic phase was dried over anhydrous sodium sulfate, filtered and concentrated to dryness, thus yielding the corresponding pyrrolidine $6 a-i$.

2-Benzoyl-N-cyclohexyl-1-(2-nitrobenzoyl)pyrrolidine-2-carboxamide (6a). Light brown solid. Yield: 386 mg, 86\%. M. p. $133-134{ }^{\circ} \mathrm{C} .{ }^{1} \mathrm{H}$ NMR $\left(300 \mathrm{MHz}, \mathrm{CDCl}_{3}\right) \delta: 8.33$ (d, J = 7.8 Hz, $\left.1 \mathrm{H}\right), 8.15$ (d, $J=8.3 \mathrm{~Hz}, 1 \mathrm{H}), 7.86(\mathrm{~d}, J=8.3 \mathrm{~Hz}, 2 \mathrm{H}), 7.73(\mathrm{t}, J=7.5 \mathrm{~Hz}, 1 \mathrm{H}), 7.66-7.44(\mathrm{~m}, 3 \mathrm{H}), 7.37(\mathrm{t}, J=$ 7.5 Hz, $2 \mathrm{H}), 3.73-3.65(\mathrm{~m}, 1 \mathrm{H}), 3.42-3.14(\mathrm{~m}, 3 \mathrm{H}), 2.13-1.77(\mathrm{~m}, 4 \mathrm{H}), 1.54-1.00(\mathrm{~m}, 8 \mathrm{H}), 0.79-$ $0.68(\mathrm{~m}, 1 \mathrm{H}) .{ }^{13} \mathrm{C}$ NMR $\{$ DEPT-135\} (75 MHz, CDCl $)$ ) $\delta: 199.2$ (Cq), 171.9 (Cq), $171.2(\mathrm{Cq}), 148.4$ (Cq), $140.1(\mathrm{Cq}), 139.1\left(\mathrm{CH}_{\mathrm{Ar}}\right), 137.1(\mathrm{Cq}), 136.9\left(\mathrm{CH}_{\mathrm{Ar}}\right), 134.4\left(\mathrm{CH}_{\mathrm{Ar}}\right), 132.5\left(\mathrm{CH}_{\mathrm{Ar}}\right), 132.3\left(\mathrm{CH}_{\mathrm{Ar}}\right)$, 132.2 $\left(\mathrm{CH}_{\mathrm{Ar}}\right), 128.9\left(\mathrm{CH}_{\mathrm{Ar}}\right), 82.8(\mathrm{Cq}), 55.3\left(\mathrm{CH}_{2}\right), 52.6(\mathrm{CH}), 40.2\left(\mathrm{CH}_{2}\right), 36.0\left(\mathrm{CH}_{2}\right), 35.7\left(\mathrm{CH}_{2}\right)$, $29.6\left(\mathrm{CH}_{2}\right), 29.0\left(\mathrm{CH}_{2}\right), 28.5\left(\mathrm{CH}_{2}\right), 28.2\left(\mathrm{CH}_{2}\right)$. HRMS (ESI-QTOF) $\mathrm{m} / \mathrm{z}$ : calculated for $\mathrm{C}_{25} \mathrm{H}_{28} \mathrm{~N}_{3} \mathrm{O}_{5}$ $\left[\mathrm{M}+\mathrm{H}^{+}\right]$450.2023; found 450.2019.

N-Cyclohexyl-2-(4-methylbenzoyl)-1-(2-nitrobenzoyl)pyrrolidine-2-carboxamide (6b). Light brown solid. Yield: $412 \mathrm{mg}, 89 \%$. M. p. $112-114{ }^{\circ} \mathrm{C} .{ }^{1} \mathrm{H}$ NMR $\left(300 \mathrm{MHz}, \mathrm{CDCl}_{3}\right)$ $\delta: 8.39$ (d, J = 7.4 
$\mathrm{Hz}, 1 \mathrm{H}), 8.14(\mathrm{~d}, J=8.3 \mathrm{~Hz}, 1 \mathrm{H}), 7.79(\mathrm{~d}, J=8.3 \mathrm{~Hz}, 2 \mathrm{H}), 7.73-7.51(\mathrm{~m}, 3 \mathrm{H}), 7.16(\mathrm{~d}, J=8.3 \mathrm{~Hz}$, $2 \mathrm{H}), 3.80-3.64(\mathrm{~m}, 1 \mathrm{H}), 3.39-3.21(\mathrm{~m}, 3 \mathrm{H}), 2.32(\mathrm{~s}, 3 \mathrm{H}), 2.11-1.00(\mathrm{~m}, 12 \mathrm{H}), 0.84-0.75(\mathrm{~m}, 1 \mathrm{H})$. ${ }^{13}$ C NMR \{DEPT-135\} (75 MHz, CDCl $)$ 8: 193.9 (Cq), 167.7 (Cq), 167.1 (Cq), 144.1 (Cq), 143.7 (Cq), $134.9\left(\mathrm{CH}_{\mathrm{Ar}}\right), 132.9(\mathrm{Cq}), 130.2\left(\mathrm{CH}_{\mathrm{Ar}}\right), 128.9\left(\mathrm{CH}_{\mathrm{Ar}}\right), 128.4\left(\mathrm{CH}_{\mathrm{Ar}}\right), 128.0\left(\mathrm{CH}_{\mathrm{Ar}}\right), 124.6\left(\mathrm{CH}_{\mathrm{Ar}}\right)$, $78.6(\mathrm{Cq}), 51.2\left(\mathrm{CH}_{2}\right), 48.4(\mathrm{CH}), 36.0\left(\mathrm{CH}_{2}\right), 31.8\left(\mathrm{CH}_{2}\right), 31.6\left(\mathrm{CH}_{2}\right), 25.4\left(\mathrm{CH}_{2}\right), 24.7\left(\mathrm{CH}_{2}\right), 24.2$ $\left(\mathrm{CH}_{2}\right), 24.0\left(\mathrm{CH}_{2}\right), 21.6\left(\mathrm{CH}_{3}\right)$. HRMS (ESI-QTOF) $\mathrm{m} / \mathrm{z}$ : calculated for $\mathrm{C}_{26} \mathrm{H}_{30} \mathrm{~N}_{3} \mathrm{O}_{5}\left[\mathrm{M}+\mathrm{H}^{+}\right]$ 464.2180; found 464.2177.

2-(4-Chlorobenzoyl)-N-cyclohexyl-1-(2-nitrobenzoyl)pyrrolidine-2-carboxamide (6c). Light

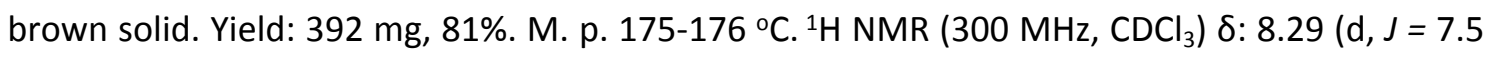
$\mathrm{Hz}, 1 \mathrm{H}), 8.13(\mathrm{~d}, J=8.3 \mathrm{~Hz}, 1 \mathrm{H}), 7.80(\mathrm{~d}, J=8.3 \mathrm{~Hz}, 2 \mathrm{H}$ ), 7.71 (app. td, ddd, $J=7.5,7.5,1.8 \mathrm{~Hz}$, $1 \mathrm{H}), 7.58-7.51(\mathrm{~m}, 2 \mathrm{H}), 7.31(\mathrm{~d}, J=8.3 \mathrm{~Hz}, 2 \mathrm{H}), 3.71-3.59(\mathrm{~m}, 1 \mathrm{H}), 3.35-3.21(\mathrm{~m}, 3 \mathrm{H}), 2.26-0.69$

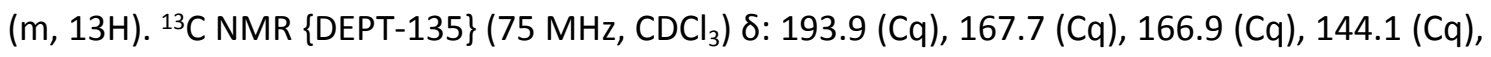
$139.1(\mathrm{Cq}), 134.9\left(\mathrm{CH}_{\mathrm{Ar}}\right), 134.2(\mathrm{Cq}), 132.6(\mathrm{Cq}), 130.3\left(\mathrm{CH}_{\mathrm{Ar}}\right), 129.7\left(\mathrm{CH}_{\mathrm{Ar}}\right), 128.5\left(\mathrm{CH}_{\mathrm{Ar}}\right), 127.9$ $\left(\mathrm{CH}_{\mathrm{Ar}}\right), 124.7\left(\mathrm{CH}_{\mathrm{Ar}}\right), 78.5(\mathrm{Cq}), 51.0\left(\mathrm{CH}_{2}\right), 48.5(\mathrm{CH}), 36.2\left(\mathrm{CH}_{2}\right), 31.8\left(\mathrm{CH}_{2}\right), 31.6\left(\mathrm{CH}_{2}\right), 25.4$ $\left(\mathrm{CH}_{2}\right), 24.7\left(\mathrm{CH}_{2}\right), 24.2\left(\mathrm{CH}_{2}\right), 24.0\left(\mathrm{CH}_{2}\right)$. HRMS (ESI-QTOF) $\mathrm{m} / \mathrm{z}$ : calculated for $\mathrm{C}_{25} \mathrm{H}_{27} \mathrm{ClN}_{3} \mathrm{O}_{5}$ $\left[\mathrm{M}+\mathrm{H}^{+}\right]$484.1634; found 484.1631.

2-Benzoyl-N-(tert-butyl)-1-(2-nitrobenzoyl)pyrrolidine-2-carboxamide (6d). Light brown solid. Yield: $339 \mathrm{mg}, 80 \%$. M. p. $106-107{ }^{\circ} \mathrm{C} .{ }^{1} \mathrm{H}$ NMR $\left(300 \mathrm{MHz}, \mathrm{CDCl}_{3}\right) \delta: 8.35(\mathrm{~s}, 1 \mathrm{H}), 8.20(\mathrm{dd}, J=$ 8.3, $1.2 \mathrm{~Hz}, 1 \mathrm{H}$ ), 7.92-7.89 (m, 2H), 7.76 (app. td, ddd, $J=7.5,7.5,1.2 \mathrm{~Hz}, 1 \mathrm{H}), 7.65-7.49(\mathrm{~m}$, $3 \mathrm{H}), 7.40(\mathrm{t}, J=7.5 \mathrm{~Hz}, 2 \mathrm{H}), 3.49-3.17(\mathrm{~m}, 3 \mathrm{H}), 2.16-1.92(\mathrm{~m}, 3 \mathrm{H}), 1.16(\mathrm{~s}, 9 \mathrm{H}) .{ }^{13} \mathrm{C}$ NMR \{DEPT$135\}\left(75 \mathrm{MHz}, \mathrm{CDCl}_{3}\right)$ ס: $194.9(\mathrm{Cq}), 167.6(\mathrm{Cq}), 166.5(\mathrm{Cq}), 144.2(\mathrm{Cq}), 135.9(\mathrm{Cq}), 134.8\left(\mathrm{CH}_{\mathrm{Ar}}\right)$, $132.9(\mathrm{Cq}), 132.7\left(\mathrm{CH}_{\mathrm{Ar}}\right), 130.2\left(\mathrm{CH}_{\mathrm{Ar}}\right), 128.2\left(\mathrm{CH}_{\mathrm{Ar}}\right), 128.2\left(\mathrm{CH}_{\mathrm{Ar}}\right), 128.0\left(\mathrm{CH}_{\mathrm{Ar}}\right), 124.7\left(\mathrm{CH}_{\mathrm{Ar}}\right), 79.2$ (Cq), $51.3\left(\mathrm{CH}_{2}\right), 51.2(\mathrm{Cq}), 35.9\left(\mathrm{CH}_{2}\right), 27.9\left(\mathrm{CH}_{3}\right), 24.8\left(\mathrm{CH}_{2}\right)$. HRMS (ESI-QTOF) m/z: calculated for $\mathrm{C}_{23} \mathrm{H}_{26} \mathrm{~N}_{3} \mathrm{O}_{5}\left[\mathrm{M}+\mathrm{H}^{+}\right]$424.1867; found 424.1864. 
2-Benzoyl-N-butyl-1-(2-nitrobenzoyl)pyrrolidine-2-carboxamide (6e). Light brown solid. Yield: $368 \mathrm{mg}, 87 \%$. M. p. $107-108{ }^{\circ} \mathrm{C} .{ }^{1} \mathrm{H}$ NMR $\left(300 \mathrm{MHz}, \mathrm{CDCl}_{3}\right) \delta: 8.25(\mathrm{t}, J=5.1 \mathrm{~Hz}, 1 \mathrm{H}), 8.11(\mathrm{~d}, J=$ $8.0 \mathrm{~Hz}, 1 \mathrm{H}$ ), 7.83 (d, $J=7.5 \mathrm{~Hz}, 2 \mathrm{H}), 7.69$ (app. td, ddd, $J=7.5,7.5,1.2 \mathrm{~Hz}, 1 \mathrm{H}), 7.54-7.42$ (m, $3 \mathrm{H}), 7.34(\mathrm{t}, J=7.5 \mathrm{~Hz}, 2 \mathrm{H}), 3.38-3.02(\mathrm{~m}, 5 \mathrm{H}), 2.09-1.87(\mathrm{~m}, 3 \mathrm{H}), 1.26-1.17(\mathrm{~m}, 2 \mathrm{H}), 1.05-0.92$ $(\mathrm{m}, 2 \mathrm{H}), 0.65(\mathrm{t}, J=7.2 \mathrm{~Hz}, 3 \mathrm{H}) .{ }^{13} \mathrm{C}$ NMR $\left\{\right.$ DEPT-135\} $\left(75 \mathrm{MHz}, \mathrm{CDCl}_{3}\right) \delta: 194.8(\mathrm{Cq}), 168.1(\mathrm{Cq})$, $167.6(\mathrm{Cq}), 144.2(\mathrm{Cq}), 135.8(\mathrm{Cq}), 134.9\left(\mathrm{CH}_{\mathrm{Ar}}\right), 132.8\left(\mathrm{CH}_{\mathrm{Ar}}\right), 132.6(\mathrm{Cq}), 130.3\left(\mathrm{CH}_{\mathrm{Ar}}\right), 128.3$ $\left(\mathrm{CH}_{\mathrm{Ar}}\right), 128.1\left(\mathrm{CH}_{\mathrm{Ar}}\right), 127.8\left(\mathrm{CH}_{\mathrm{Ar}}\right), 124.7\left(\mathrm{CH}_{\mathrm{Ar}}\right), 78.5(\mathrm{Cq}), 51.0\left(\mathrm{CH}_{2}\right), 39.7\left(\mathrm{CH}_{2}\right), 36.0\left(\mathrm{CH}_{2}\right), 30.7$ $\left(\mathrm{CH}_{2}\right), 24.7\left(\mathrm{CH}_{2}\right), 19.8\left(\mathrm{CH}_{2}\right), 13.6\left(\mathrm{CH}_{3}\right)$. HRMS (ESI-QTOF) m/z: calculated for $\mathrm{C}_{23} \mathrm{H}_{26} \mathrm{~N}_{3} \mathrm{O}_{5}$ $\left[\mathrm{M}+\mathrm{H}^{+}\right]$424.1867; found 424.1865.

2-Benzoyl-1-(5-chloro-2-nitrobenzoyl)-N-cyclohexylpyrrolidine-2-carboxamide (6f). Light

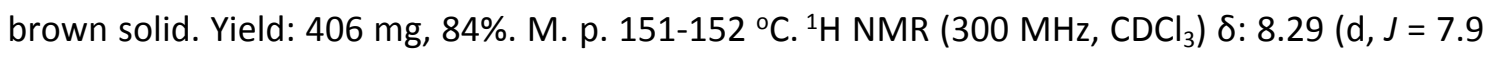
$\mathrm{Hz}, 1 \mathrm{H}), 8.19(\mathrm{~d}, J=8.8 \mathrm{~Hz}, 1 \mathrm{H}), 7.92(\mathrm{~d}, J=7.3 \mathrm{~Hz}, 2 \mathrm{H}), 7.68(\mathrm{~d}, J=2.3 \mathrm{~Hz}, 1 \mathrm{H}), 7.58-7.53(\mathrm{~m}$, $2 \mathrm{H}), 7.44(\mathrm{t}, J=7.8 \mathrm{~Hz}, 2 \mathrm{H}), 3.72-3.64(\mathrm{~m}, 1 \mathrm{H}), 3.44-3.25(\mathrm{~m}, 3 \mathrm{H}), 2.14-1.92(\mathrm{~m}, 3 \mathrm{H}), 1.77-0.67$ (m, 10H). ${ }^{13} \mathrm{C}$ NMR $\{$ DEPT-135\} (75 MHz, CDCl $)$ ) $8: 194.6(\mathrm{Cq}), 166.6(\mathrm{Cq}), 166.2(\mathrm{Cq}), 142.5(\mathrm{Cq})$, $141.6(\mathrm{Cq}), 135.6(\mathrm{Cq}), 134.3(\mathrm{Cq}), 132.9\left(\mathrm{CH}_{\mathrm{Ar}}\right), 130.3\left(\mathrm{CH}_{\mathrm{Ar}}\right), 128.3\left(\mathrm{CH}_{\mathrm{Ar}}\right), 128.1\left(\mathrm{CH}_{\mathrm{Ar}}\right), 128.0$ $\left(\mathrm{CH}_{\mathrm{Ar}}\right), 126.3\left(\mathrm{CH}_{\mathrm{Ar}}\right), 78.7(\mathrm{Cq}), 51.1\left(\mathrm{CH}_{2}\right), 48.4(\mathrm{CH}), 36.0\left(\mathrm{CH}_{2}\right), 31.8\left(\mathrm{CH}_{2}\right), 31.5\left(\mathrm{CH}_{2}\right), 25.4$ $\left(\mathrm{CH}_{2}\right), 24.8\left(\mathrm{CH}_{2}\right), 24.2\left(\mathrm{CH}_{2}\right), 23.9\left(\mathrm{CH}_{2}\right)$. HRMS (ESI-QTOF) $\mathrm{m} / \mathrm{z}$ : calculated for $\mathrm{C}_{25} \mathrm{H}_{27} \mathrm{ClN}_{3} \mathrm{O}_{5}$ $\left[\mathrm{M}+\mathrm{H}^{+}\right]$484.1634; found 484.1629 .

\section{1-(4-Chloro-2-nitrobenzoyl)-N-cyclohexyl-2-(4-methylbenzoyl)pyrrolidine-2-carboxamide}

(6g). Light brown solid. Yield: $433 \mathrm{mg}, 87 \%$. M. p. $135-136^{\circ} \mathrm{C} .{ }^{1} \mathrm{H}$ NMR $\left(300 \mathrm{MHz}, \mathrm{CDCl}_{3}\right)$ 8: 8.35 (d, $J=7.8 \mathrm{~Hz}, 1 \mathrm{H}), 8.19(\mathrm{~d}, J=2.0 \mathrm{~Hz}, 1 \mathrm{H}), 7.83(\mathrm{~d}, J=8.0 \mathrm{~Hz}, 2 \mathrm{H}), 7.74(\mathrm{dd}, J=8.2,2.0 \mathrm{~Hz}, 1 \mathrm{H}$ ), $7.65(\mathrm{~d}, J=8.2 \mathrm{~Hz}, 1 \mathrm{H}), 7.20(\mathrm{~d}, J=8.0 \mathrm{~Hz}, 2 \mathrm{H}), 3.79-3.67(\mathrm{~m}, 1 \mathrm{H}), 3.46-3.26(\mathrm{~m}, 3 \mathrm{H}), 2.37(\mathrm{~s}$,

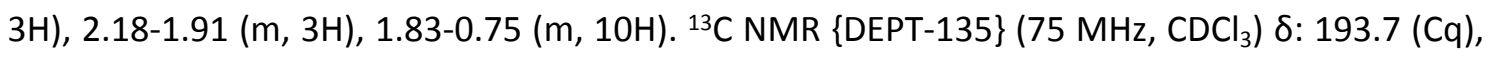
$166.8(\mathrm{Cq}), 166.8(\mathrm{Cq}), 144.8(\mathrm{Cq}), 143.9(\mathrm{Cq}), 136.1(\mathrm{Cq}), 134.9\left(\mathrm{CH}_{\mathrm{Ar}}\right), 132.7(\mathrm{Cq}), 131.4(\mathrm{Cq})$, 129.3 $\left(\mathrm{CH}_{\mathrm{Ar}}\right), 129.0\left(\mathrm{CH}_{\mathrm{Ar}}\right), 128.5\left(\mathrm{CH}_{\mathrm{Ar}}\right), 124.9\left(\mathrm{CH}_{\mathrm{Ar}}\right), 78.8(\mathrm{Cq}), 51.3\left(\mathrm{CH}_{2}\right), 48.5(\mathrm{CH}), 36.1\left(\mathrm{CH}_{2}\right)$, 
31.8 $\left(\mathrm{CH}_{2}\right), 31.6\left(\mathrm{CH}_{2}\right), 25.4\left(\mathrm{CH}_{2}\right), 24.8\left(\mathrm{CH}_{2}\right), 24.3\left(\mathrm{CH}_{2}\right), 24.0\left(\mathrm{CH}_{2}\right), 21.7\left(\mathrm{CH}_{3}\right)$. HRMS (ESIQTOF) $m / z$ : calculated for $\mathrm{C}_{26} \mathrm{H}_{29} \mathrm{ClN}_{3} \mathrm{O}_{5}\left[\mathrm{M}+\mathrm{H}^{+}\right]$498.1790; found 498.1781 .

2-Benzoyl-N-(tert-butyl)-1-(4-chloro-2-nitrobenzoyl)pyrrolidine-2-carboxamide (6h). Light brown solid. Yield: $348 \mathrm{mg}, 76 \%$. M. p. $123-124{ }^{\circ} \mathrm{C} .{ }^{1} \mathrm{H}$ NMR $\left(300 \mathrm{MHz}, \mathrm{CDCl}_{3}\right) \delta: 8.26(\mathrm{~s}, 1 \mathrm{H})$, $8.15(\mathrm{~d}, J=2.0 \mathrm{~Hz}, 1 \mathrm{H}), 7.88-7.85(\mathrm{~m}, 2 \mathrm{H}), 7.71(\mathrm{dd}, J=8.2,2.0 \mathrm{~Hz}, 1 \mathrm{H}), 7.58(\mathrm{~d}, J=8.2 \mathrm{~Hz}, 1 \mathrm{H})$, $7.49(\mathrm{tt}, J=7.2,2.0 \mathrm{~Hz}, 1 \mathrm{H}), 7.37(\mathrm{t}, J=7.2 \mathrm{~Hz}, 2 \mathrm{H}), 3.41-3.22(\mathrm{~m}, 3 \mathrm{H}), 2.14-1.91(\mathrm{~m}, 3 \mathrm{H}), 1.12$

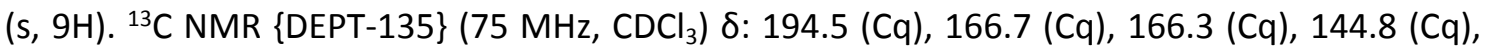
$136.1(\mathrm{Cq}), 135.6(\mathrm{Cq}), 134.9\left(\mathrm{CH}_{\mathrm{Ar}}\right), 132.8\left(\mathrm{CH}_{\mathrm{Ar}}\right), 131.2(\mathrm{Cq}), 129.2\left(\mathrm{CH}_{\mathrm{Ar}}\right), 128.3\left(\mathrm{CH}_{\mathrm{Ar}}\right), 128.2$ $\left(\mathrm{CH}_{\mathrm{Ar}}\right), 124.9\left(\mathrm{CH}_{\mathrm{Ar}}\right), 79.3(\mathrm{Cq}), 51.3(\mathrm{Cq}), 51.2\left(\mathrm{CH}_{2}\right), 35.9\left(\mathrm{CH}_{2}\right), 27.9\left(\mathrm{CH}_{3}\right), 24.8\left(\mathrm{CH}_{2}\right)$. HRMS (ESI-QTOF) $\mathrm{m} / \mathrm{z}$ : calculated for $\mathrm{C}_{23} \mathrm{H}_{25} \mathrm{ClN}_{3} \mathrm{O}_{5}\left[\mathrm{M}+\mathrm{H}^{+}\right]$458.1477; found 458.1475 .

\section{1-(4-Chloro-2-nitrobenzoyl)-N-cyclohexyl-2-(4-methoxybenzoyl)pyrrolidine-2-carboxamide}

(6i). Light brown solid. Yield: $442 \mathrm{mg}, 86 \%$. M. p. $112-113{ }^{\circ} \mathrm{C} .{ }^{1} \mathrm{H}$ NMR $\left(300 \mathrm{MHz}, \mathrm{CDCl}_{3}\right)$ 8: 8.45 (d, $J=7.3 \mathrm{~Hz}, 1 \mathrm{H}), 8.19(\mathrm{~s}, 1 \mathrm{H}), 7.98-7.94(\mathrm{~m}, 2 \mathrm{H}), 7.77-7.67(\mathrm{~m}, 2 \mathrm{H}), 6.96-6.84(\mathrm{~m}, 2 \mathrm{H}), 3.84(\mathrm{~s}$, $3 \mathrm{H}), 3.82-3.69(\mathrm{~m}, 1 \mathrm{H}), 3.45-3.29(\mathrm{~m}, 3 \mathrm{H}), 2.21-1.93(\mathrm{~m}, 3 \mathrm{H}), 1.86-0.81(\mathrm{~m}, 10 \mathrm{H}) .{ }^{13} \mathrm{C} N M R$ \{DEPT-135\} (75 MHz, CDCl $)$ ) ס: $191.9(\mathrm{Cq}), 167.0(\mathrm{Cq}), 166.9(\mathrm{Cq}), 163.5(\mathrm{Cq}), 144.8(\mathrm{Cq}), 136.0$ (Cq), $134.9\left(\mathrm{CH}_{\mathrm{Ar}}\right), 131.5(\mathrm{Cq}), 131.1\left(\mathrm{CH}_{\mathrm{Ar}}\right), 129.4\left(\mathrm{CH}_{\mathrm{Ar}}\right), 127.8(\mathrm{Cq}), 124.9\left(\mathrm{CH}_{\mathrm{Ar}}\right), 113.6\left(\mathrm{CH}_{\mathrm{Ar}}\right)$, $78.8(\mathrm{Cq}), 55.5\left(\mathrm{CH}_{3}\right), 51.4\left(\mathrm{CH}_{2}\right), 48.5(\mathrm{CH}), 36.1\left(\mathrm{CH}_{2}\right), 31.9\left(\mathrm{CH}_{2}\right), 31.8\left(\mathrm{CH}_{2}\right), 25.4\left(\mathrm{CH}_{2}\right), 24.7$ $\left(\mathrm{CH}_{2}\right), 24.3\left(\mathrm{CH}_{2}\right), 24.1\left(\mathrm{CH}_{2}\right)$. HRMS (ESI-QTOF) $\mathrm{m} / \mathrm{z}$ : calculated for $\mathrm{C}_{26} \mathrm{H}_{29} \mathrm{ClN}_{3} \mathrm{O}_{6}\left[\mathrm{M}+\mathrm{H}^{+}\right]$ 514.1739; found 514.1737.

General procedure for the synthesis of 3-pyrrolines 7a-f. A mixture of the corresponding Ugi adduct 5j-o (1.0 mmol, 1.0 equiv., $0.1 \mathrm{M})$ and $\mathrm{Na}_{2} \mathrm{CO}_{3}(1.0 \mathrm{mmol}, 1.0$ equiv.) in boiling ethanol $(10 \mathrm{~mL})$ was stirred for 6 hours, after which the solvent was removed by rotary evaporation. The raw product was dissolved in dichloromethane and washed with acidified water. The organic phase was dried over anhydrous sodium sulfate, filtered and concentrated to dryness, thus yielding the corresponding 3-pyrrolines 7a-f. 
2-Benzoyl-N-cyclohexyl-1-(2-nitrobenzoyl)-2,5-dihydro-1H-pyrrole-2-carboxamide (7a). Sticky brown solid. Yield: $362 \mathrm{mg}, 81 \% .{ }^{1} \mathrm{H}$ NMR $\left(300 \mathrm{MHz}, \mathrm{CDCl}_{3}\right)$ 8: 8.23-8.20 (m, 2H), 7.96-7.92 (m, 2H), $7.71(\mathrm{tt}, J=7.5,1.2 \mathrm{~Hz}, 1 \mathrm{H}), 7.62-7.55(\mathrm{~m}, 2 \mathrm{H}), 7.47-7.42(\mathrm{~m}, 2 \mathrm{H}), 7.20(\mathrm{~d}, J=7.5 \mathrm{~Hz}, 1 \mathrm{H})$, $6.33(\mathrm{dt}, J=6.4,2.3 \mathrm{~Hz}, 1 \mathrm{H}), 6.04(\mathrm{dt}, J=6.4,2.3 \mathrm{~Hz}, 1 \mathrm{H}), 4.19-4.07(\mathrm{~m}, 2 \mathrm{H}), 3.88-3.77(\mathrm{~m}, 1 \mathrm{H})$,

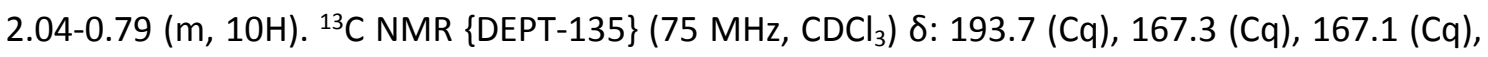
$144.2(\mathrm{Cq}), 136.8(\mathrm{Cq}), 135.0\left(\mathrm{CH}_{\mathrm{Ar}}\right), 132.6\left(\mathrm{CH}_{\mathrm{Ar}}\right), 132.1(\mathrm{Cq}), 130.5\left(\mathrm{CH}_{\mathrm{Ar}}\right), 129.6(\mathrm{CH}), 128.3$ $\left(\mathrm{CH}_{\mathrm{Ar}}\right), 128.2\left(\mathrm{CH}_{\mathrm{Ar}}\right), 127.8\left(\mathrm{CH}_{\mathrm{Ar}}\right), 127.1(\mathrm{CH}), 124.7\left(\mathrm{CH}_{\mathrm{Ar}}\right), 84.9(\mathrm{Cq}), 56.3\left(\mathrm{CH}_{2}\right), 48.5(\mathrm{CH}), 32.1$ $\left(\mathrm{CH}_{2}\right), 32.0\left(\mathrm{CH}_{2}\right), 25.5\left(\mathrm{CH}_{2}\right), 24.4\left(\mathrm{CH}_{2}\right), 24.3\left(\mathrm{CH}_{2}\right)$. HRMS (ESI-QTOF) m/z: calculated for $\mathrm{C}_{25} \mathrm{H}_{26} \mathrm{~N}_{3} \mathrm{O}_{5}\left[\mathrm{M}+\mathrm{H}^{+}\right]$448.1867; found 448.1872 .

\section{$\mathrm{N}$-Cyclohexyl-2-(4-methylbenzoyl)-1-(2-nitrobenzoyl)-2,5-dihydro-1H-pyrrole-2-carboxamide}

(7b). Sticky brown solid. Yield: $337 \mathrm{mg}, 73 \% .{ }^{1} \mathrm{H}$ NMR (300 MHz, $\left.\mathrm{CDCl}_{3}\right) \delta: 8.25$ (d, J= $7.8 \mathrm{~Hz}$, $1 \mathrm{H}), 8.19(\mathrm{~d}, J=8.3 \mathrm{~Hz}, 1 \mathrm{H}), 7.86(\mathrm{~d}, J=8.3 \mathrm{~Hz}, 2 \mathrm{H}), 7.70$ (app. td, ddd, $J=7.8,7.8,1.5 \mathrm{~Hz}, 1 \mathrm{H}$ ), 7.57 (app. td, ddd, $J=7.8,7.8,1.5 \mathrm{~Hz}, 1 \mathrm{H}$ ), 7.26 (dd, $J=7.8,1.2 \mathrm{~Hz}, 1 \mathrm{H}$ ), $7.22(\mathrm{~d}, J=8.3 \mathrm{~Hz}, 2 \mathrm{H}$ ), $6.27(\mathrm{dt}, J=6.4,2.1 \mathrm{~Hz}, 1 \mathrm{H}), 5.98(\mathrm{dt}, J=6.4,2.1 \mathrm{~Hz}, 1 \mathrm{H}), 4.10(\mathrm{t}, J=2.1 \mathrm{~Hz}, 2 \mathrm{H}), 3.86-3.74(\mathrm{~m}$, 1H), 2.39 (s, 3H), 2.05-0.76 (m, 10H). ${ }^{13} \mathrm{C}$ NMR $\left\{\right.$ DEPT-135\} (75 MHz, $\mathrm{CDCl}_{3}$ ) $\delta: 192.4(\mathrm{Cq}), 167.4$ (Cq), $167.3(\mathrm{Cq}), 144.2(\mathrm{Cq}), 143.7(\mathrm{Cq}), 135.0(\mathrm{CH}), 133.5(\mathrm{Cq}), 132.3(\mathrm{Cq}), 130.4(\mathrm{CH}), 129.7$ (CH), $129.0(\mathrm{CH}), 128.6(\mathrm{CH}), 128.0(\mathrm{CH}), 126.8(\mathrm{CH}), 124.7(\mathrm{CH}), 85.1(\mathrm{Cq}), 56.4\left(\mathrm{CH}_{2}\right), 48.5$ (CH), $32.1\left(\mathrm{CH}_{2}\right), 32.0\left(\mathrm{CH}_{2}\right), 25.5\left(\mathrm{CH}_{2}\right), 24.4\left(\mathrm{CH}_{2}\right), 24.4\left(\mathrm{CH}_{2}\right), 21.7\left(\mathrm{CH}_{3}\right)$. HRMS (ESI-QTOF) $\mathrm{m} / \mathrm{z}$ : calculated for $\mathrm{C}_{26} \mathrm{H}_{28} \mathrm{~N}_{3} \mathrm{O}_{5}\left[\mathrm{M}+\mathrm{H}^{+}\right]$462.2023; found 462.2036.

\section{N-Cyclohexyl-2-(4-methoxybenzoyl)-1-(2-nitrobenzoyl)-2,5-dihydro-1H-pyrrole-2-}

carboxamide (7c). Sticky brown solid. Yield: $391 \mathrm{mg}, 82 \% .{ }^{1} \mathrm{H} \mathrm{NMR}\left(300 \mathrm{MHz}, \mathrm{CDCl}_{3}\right)$ 8: 8.35 (d, $J=7.5 \mathrm{~Hz}, 1 \mathrm{H}), 8.19(\mathrm{~d}, J=8.3 \mathrm{~Hz}, 1 \mathrm{H}), 8.00(\mathrm{~d}, J=8.3 \mathrm{~Hz}, 2 \mathrm{H}), 7.72(\mathrm{t}, J=7.5 \mathrm{~Hz}, 1 \mathrm{H}), 7.57(\mathrm{t}, J=$ $7.5 \mathrm{~Hz}, 1 \mathrm{H}), 7.42(\mathrm{~d}, J=7.5 \mathrm{~Hz}, 1 \mathrm{H}), 6.89(\mathrm{~d}, J=8.3 \mathrm{~Hz}, 2 \mathrm{H}), 6.25(\mathrm{~d}, J=6.3 \mathrm{~Hz}, 1 \mathrm{H}), 5.94(\mathrm{~d}, J=$ $6.3 \mathrm{~Hz}, 1 \mathrm{H}), 4.09(\mathrm{~s}, 2 \mathrm{H}), 3.90-3.73(\mathrm{~m}, 1 \mathrm{H}), 3.83(\mathrm{~s}, 3 \mathrm{H}), 2.07-0.73(\mathrm{~m}, 10 \mathrm{H}) .{ }^{13} \mathrm{C}$ NMR \{DEPT-

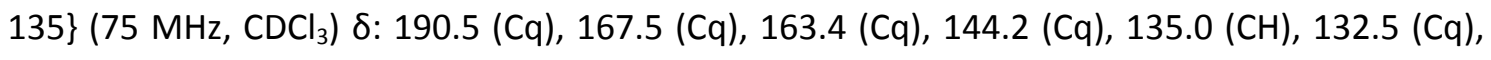
$131.1(\mathrm{CH}), 130.4(\mathrm{CH}), 129.7(\mathrm{CH}), 128.3(\mathrm{Cq}), 128.1(\mathrm{CH}), 126.5(\mathrm{CH}), 124.7(\mathrm{CH}), 113.6(\mathrm{CH})$, 
$85.1(\mathrm{Cq}), 56.5\left(\mathrm{CH}_{2}\right), 55.5\left(\mathrm{CH}_{3}\right), 48.5(\mathrm{CH}), 32.0\left(\mathrm{CH}_{2}\right), 25.5\left(\mathrm{CH}_{2}\right), 24.4\left(\mathrm{CH}_{2}\right), 24.3\left(\mathrm{CH}_{2}\right)$. HRMS (ESI-QTOF) $\mathrm{m} / \mathrm{z}$ : calculated for $\mathrm{C}_{26} \mathrm{H}_{28} \mathrm{~N}_{3} \mathrm{O}_{6}\left[\mathrm{M}+\mathrm{H}^{+}\right]$478.1973; found 478.1976 .

\section{2-Benzoyl-N-(tert-butyl)-1-(2-nitrobenzoyl)-2,5-dihydro-1H-pyrrole-2-carboxamide}

(7d).

Sticky brown solid. Yield: $303 \mathrm{mg}, 72 \% .{ }^{1} \mathrm{H}$ NMR $\left(300 \mathrm{MHz}, \mathrm{CDCl}_{3}\right) \delta: 8.22(\mathrm{~s}, 1 \mathrm{H}), 8.18$ (dd, $\mathrm{J}=$ 8.2, $1.2 \mathrm{~Hz}, 1 \mathrm{H}$ ), 7.92 (d, $J=7.5 \mathrm{~Hz}, 2 \mathrm{H}$ ), 7.68 (app. td, ddd, $J=7.5,7.5,1.2 \mathrm{~Hz}, 1 \mathrm{H}$ ), 7.61-7.51 (m, 2H), $7.42(\mathrm{t}, J=7.5 \mathrm{~Hz}, 2 \mathrm{H}), 7.09(\mathrm{dd}, J=7.5,1.2 \mathrm{~Hz}, 1 \mathrm{H}), 6.27(\mathrm{dt}, J=6.3,2.2 \mathrm{~Hz}, 1 \mathrm{H}), 6.01$ $(\mathrm{dt}, J=6.3,2.2 \mathrm{~Hz}, 1 \mathrm{H}), 4.18-3.95(\mathrm{~m}, 2 \mathrm{H}), 1.30(\mathrm{~s}, 9 \mathrm{H}) .{ }^{13} \mathrm{C}$ NMR $\left\{\right.$ DEPT-135\} $\left(75 \mathrm{MHz}, \mathrm{CDCl}_{3}\right) \delta:$ $193.4(\mathrm{Cq}), 167.3(\mathrm{Cq}), 167.1(\mathrm{Cq}), 144.2(\mathrm{Cq}), 136.5(\mathrm{Cq}), 135.0(\mathrm{CH}), 132.7(\mathrm{CH}), 132.1(\mathrm{Cq})$, $130.5(\mathrm{CH}), 129.7(\mathrm{CH}), 128.3(\mathrm{CH}), 128.3(\mathrm{CH}), 127.8(\mathrm{CH}), 127.0(\mathrm{CH}), 124.7(\mathrm{CH}), 85.6(\mathrm{Cq})$, $56.4\left(\mathrm{CH}_{2}\right), 51.5(\mathrm{Cq}), 28.2\left(\mathrm{CH}_{3}\right)$. HRMS (ESI-QTOF) $\mathrm{m} / \mathrm{z}$ : calculated for $\mathrm{C}_{23} \mathrm{H}_{24} \mathrm{~N}_{3} \mathrm{O}_{5}\left[\mathrm{M}+\mathrm{H}^{+}\right]$ 422.1710; found 422.1714 .

\section{2-Benzoyl-1-(5-chloro-2-nitrobenzoyl)-N-cyclohexyl-2,5-dihydro-1H-pyrrole-2-carboxamide}

(7e). Sticky brown solid. Yield: $371 \mathrm{mg}, 77 \% .{ }^{1} \mathrm{H}$ NMR $\left(300 \mathrm{MHz}, \mathrm{CDCl}_{3}\right) \delta: 8.12$ (d, J = $8.8 \mathrm{~Hz}$, $1 \mathrm{H}), 8.10(\mathrm{~d}, J=8.8 \mathrm{~Hz}, 1 \mathrm{H}), 7.90-7.86(\mathrm{~m}, 2 \mathrm{H}), 7.60-7.40(\mathrm{~m}, 4 \mathrm{H}), 6.99(\mathrm{~s}, 1 \mathrm{H}), 6.30(\mathrm{dt}, J=6.4$, $2.1 \mathrm{~Hz}, 1 \mathrm{H}), 6.04(\mathrm{dt}, J=6.4,2.1 \mathrm{~Hz}, 1 \mathrm{H}), 4.19-4.07(\mathrm{~m}, 2 \mathrm{H}), 3.85-3.74(\mathrm{~m}, 1 \mathrm{H}), 2.00-0.73(\mathrm{~m}$, 10H). ${ }^{13} \mathrm{C}$ NMR $\{$ DEPT-135\} (75 MHz, CDCl 3 ) $\delta: 193.0(\mathrm{Cq}), 166.8$ (Cq), 165.8 (Cq), 142.4 (Cq), $141.8(\mathrm{Cq}), 136.7(\mathrm{Cq}), 133.5(\mathrm{Cq}), 132.8(\mathrm{CH}), 130.5(\mathrm{CH}), 129.6(\mathrm{CH}), 128.4(\mathrm{CH}), 128.3(\mathrm{CH})$, 128.0 (CH), $127.1(\mathrm{CH}), 126.2(\mathrm{CH}), 85.1(\mathrm{Cq}), 56.3\left(\mathrm{CH}_{2}\right), 48.5(\mathrm{CH}), 32.1\left(\mathrm{CH}_{2}\right), 32.0\left(\mathrm{CH}_{2}\right), 25.5$ $\left(\mathrm{CH}_{2}\right), 24.4\left(\mathrm{CH}_{2}\right), 24.3\left(\mathrm{CH}_{2}\right)$. HRMS (ESI-QTOF) $\mathrm{m} / \mathrm{z}$ : calculated for $\mathrm{C}_{25} \mathrm{H}_{25} \mathrm{ClN}_{3} \mathrm{O}_{5}\left[\mathrm{M}+\mathrm{H}^{+}\right]$ 482.1477; found 482.1488.

\section{2-Benzoyl-1-(4-chloro-2-nitrobenzoyl)- $\mathrm{N}$-cyclohexyl-2,5-dihydro-1H-pyrrole-2-carboxamide}

(7f). Sticky brown solid. Yield: $419 \mathrm{mg}, 87 \% .{ }^{1} \mathrm{H}$ NMR $\left(300 \mathrm{MHz}, \mathrm{CDCl}_{3}\right)$ 8: 8.20-8.07 (m, 2H), $7.87(\mathrm{~d}, J=7.5 \mathrm{~Hz}, 2 \mathrm{H}), 7.66-7.62(\mathrm{~m}, 1 \mathrm{H}), 7.53(\mathrm{t}, J=7.5 \mathrm{~Hz}, 1 \mathrm{H}), 7.40(\mathrm{t}, J=7.5 \mathrm{~Hz}, 2 \mathrm{H}), 7.10$ $(\mathrm{d}, J=8.2 \mathrm{~Hz}, 1 \mathrm{H}), 6.26(\mathrm{~d}, J=6.1 \mathrm{~Hz}, 1 \mathrm{H}), 6.02(\mathrm{~d}, J=6.1 \mathrm{~Hz}, 1 \mathrm{H}), 4.16-4.04(\mathrm{~m}, 2 \mathrm{H}), 3.83-3.71$

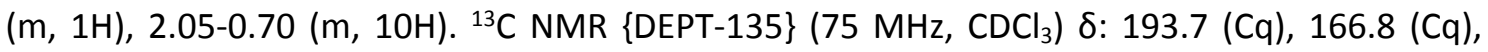


$166.3(\mathrm{Cq}), 144.7(\mathrm{Cq}), 136.6(\mathrm{Cq}), 136.4(\mathrm{Cq}), 135.0(\mathrm{CH}), 132.7(\mathrm{CH}), 130.4(\mathrm{Cq}), 129.5(\mathrm{CH})$, 129.2 (CH), $128.3(\mathrm{CH}), 128.2(\mathrm{CH}), 127.2(\mathrm{CH}), 124.8(\mathrm{CH}), 85.0(\mathrm{Cq}), 56.3\left(\mathrm{CH}_{2}\right), 48.5(\mathrm{CH}), 32.1$ $\left(\mathrm{CH}_{2}\right), 32.0\left(\mathrm{CH}_{2}\right), 25.5\left(\mathrm{CH}_{2}\right), 24.4\left(\mathrm{CH}_{2}\right), 24.3\left(\mathrm{CH}_{2}\right)$. HRMS (ESI-QTOF) m/z: calculated for $\mathrm{C}_{25} \mathrm{H}_{25} \mathrm{ClN}_{3} \mathrm{O}_{5}\left[\mathrm{M}+\mathrm{H}^{+}\right]$482.1477; found 482.1483 .

General procedure for the synthesis of 3-methylenepyrrolidines 11a-c. A mixture of the corresponding Ugi adduct 5p-r (1.0 mmol, 1.0 equiv., $0.1 \mathrm{M})$ and $\mathrm{InCl}_{3}(0.05 \mathrm{mmol}, 0.05$ equiv.) in toluene $(10 \mathrm{~mL})$ was heated to reflux for two hours. After removing the solvent by rotary evaporation, the raw product was dissolved in dichloromethane and washed with slightly basic water. The organic phase was dried over anhydrous sodium sulfate, filtered and concentrated to dryness, giving the corresponding 3-methylenepyrrolidines 11a-c.

\section{2-Benzoyl-N-cyclohexyl-3-methylene-1-(2-nitrobenzoyl)pyrrolidine-2-carboxamide}

(11a). White solid. Yield: $406 \mathrm{mg}, 88 \%$. M. p. $158-160{ }^{\circ} \mathrm{C} .{ }^{1} \mathrm{H} \mathrm{NMR}\left(300 \mathrm{MHz}, \mathrm{CDCl}_{3}\right) \delta: 8.34(\mathrm{~d}, J=7.7$ $\mathrm{Hz}, 1 \mathrm{H}, \mathrm{NH}), 8.10(\mathrm{dd}, J=8.1,1.2 \mathrm{~Hz}, 1 \mathrm{H}), 7.71-7.40(\mathrm{~m}, 7 \mathrm{H}), 7.00(\mathrm{~s}, 1 \mathrm{H}), 5.40-5.22(\mathrm{~m}, 2 \mathrm{H})$, 3.95-3.74 (m, 1H), $3.66(\mathrm{td}, J=9.9,4.8 \mathrm{~Hz}, 1 \mathrm{H}), 3.58-3.49(\mathrm{~m}, 1 \mathrm{H}), 3.20-3.07(\mathrm{~m}, 1 \mathrm{H}), 3.03-2.90$ (m, 1H), 2.00-0.82 (m, 10H). ${ }^{13} \mathrm{C}$ NMR $\{$ DEPT-135\} (75 MHz, CDCl $)$ ) : 198.7 (Cq), 166.7 (Cq), $166.6(\mathrm{Cq}), 144.9(\mathrm{Cq}), 144.4(\mathrm{Cq}), 137.3(\mathrm{Cq}), 134.4\left(\mathrm{CH}_{\mathrm{Ar}}\right), 131.9\left(\mathrm{CH}_{\mathrm{Ar}}\right), 131.8(\mathrm{Cq}), 130.1$ $\left(\mathrm{CH}_{\mathrm{Ar}}\right), 128.3\left(\mathrm{CH}_{\mathrm{Ar}}\right), 128.1\left(\mathrm{CH}_{\mathrm{Ar}}\right), 127.9\left(\mathrm{CH}_{\mathrm{Ar}}\right), 124.3\left(\mathrm{CH}_{\mathrm{Ar}}\right), 111.8\left(\mathrm{CH}_{2}\right), 78.5(\mathrm{Cq}), 48.8(\mathrm{CH})$, $47.8\left(\mathrm{CH}_{2}\right), 32.3\left(\mathrm{CH}_{2}\right), 32.2\left(\mathrm{CH}_{2}\right), 31.0\left(\mathrm{CH}_{2}\right), 25.6\left(\mathrm{CH}_{2}\right), 24.6\left(\mathrm{CH}_{2}\right), 24.5\left(\mathrm{CH}_{2}\right)$. HRMS (ESIQTOF) $m / z$ : calculated for $\mathrm{C}_{26} \mathrm{H}_{28} \mathrm{~N}_{3} \mathrm{O}_{5}\left[\mathrm{M}+\mathrm{H}^{+}\right]$462.2023; found 462.2033 .

\section{2-Benzoyl-N-(tert-butyl)-3-methylene-1-(2-nitrobenzoyl)pyrrolidine-2-carboxamide}

(11b).

Brown solid. Yield: $340 \mathrm{mg}, 78 \%$. M. p. $140-142{ }^{\circ} \mathrm{C} .{ }^{1} \mathrm{H}$ NMR $\left(300 \mathrm{MHz}, \mathrm{CDCl}_{3}\right)$ ): 8.24 (br s, $1 \mathrm{H}$, $\mathrm{NH}), 8.05(\mathrm{dd}, J=8.2,1.4 \mathrm{~Hz}, 1 \mathrm{H}), 7.69-7.36(\mathrm{~m}, 7 \mathrm{H}), 6.78(\mathrm{~d}, J=6.7 \mathrm{~Hz}, 1 \mathrm{H}), 5.32(\mathrm{~s}, 1 \mathrm{H}), 5.22$ $(\mathrm{s}, 1 \mathrm{H}), 3.61-3.45(\mathrm{~m}, 2 \mathrm{H}), 3.14-3.02(\mathrm{~m}, 1 \mathrm{H}), 2.98-2.86(\mathrm{~m}, 1 \mathrm{H}), 1.39(\mathrm{~s}, 9 \mathrm{H}) .{ }^{13} \mathrm{C}$ NMR \{DEPT135\} (75 MHz, CDCl $)$ ) ס: $198.2(\mathrm{Cq}), 166.7(\mathrm{Cq}), 166.1(\mathrm{Cq}), 144.6(\mathrm{Cq}), 144.4(\mathrm{Cq}), 137.2(\mathrm{Cq})$, $134.5\left(\mathrm{CH}_{\mathrm{Ar}}\right), 131.9\left(\mathrm{CH}_{\mathrm{Ar}}\right), 131.7(\mathrm{Cq}), 130.2\left(\mathrm{CH}_{\mathrm{Ar}}\right), 128.2\left(\mathrm{CH}_{\mathrm{Ar}}\right), 127.9\left(\mathrm{CH}_{\mathrm{Ar}}\right), 124.4\left(\mathrm{CH}_{\mathrm{Ar}}\right)$, 
$112.1\left(\mathrm{CH}_{2}\right), 79.3(\mathrm{Cq}), 51.4(\mathrm{Cq}), 47.8\left(\mathrm{CH}_{2}\right), 31.0\left(\mathrm{CH}_{2}\right), 28.4\left(\mathrm{CH}_{3}\right)$. HRMS (ESI-QTOF) $\mathrm{m} / \mathrm{z}$ : calculated for $\mathrm{C}_{24} \mathrm{H}_{26} \mathrm{~N}_{3} \mathrm{O}_{5}\left[\mathrm{M}+\mathrm{H}^{+}\right]$436.1867; found 436.1877 .

\section{2-Benzoyl-1-(4-chloro-2-nitrobenzoyl)-N-cyclohexyl-3-methylenepyrrolidine-2-carboxamide} (11c). White solid. Yield: $347 \mathrm{mg}, 70 \%$. M. p. $180-182{ }^{\circ} \mathrm{C} .{ }^{1} \mathrm{H}$ NMR $\left(300 \mathrm{MHz}, \mathrm{CDCl}_{3}\right) \delta: 8.37(\mathrm{~d}, J$ $=6.8 \mathrm{~Hz}, 1 \mathrm{H}, \mathrm{NH}), 8.06-8.04(\mathrm{~m}, 1 \mathrm{H}), 7.66-7.36(\mathrm{~m}, 6 \mathrm{H}), 6.99(\mathrm{~d}, J=8.3 \mathrm{~Hz}, 1 \mathrm{H}), 5.39-5.20(\mathrm{~m}$, $2 \mathrm{H}), 3.87-3.77(\mathrm{~m}, 1 \mathrm{H}), 3.68-3.60(\mathrm{~m}, 1 \mathrm{H}), 3.56-3.47(\mathrm{~m}, 1 \mathrm{H}), 3.19-3.06(\mathrm{~m}, 1 \mathrm{H}), 3.04-2.91(\mathrm{~m}$, 1H), 1.99-1.23 (m, 10H). ${ }^{13} \mathrm{C}$ NMR $\{$ DEPT-135\} (75 MHz, CDCl $)$ 8: $198.9(\mathrm{Cq}), 166.6(\mathrm{Cq}), 165.7$ (Cq), $145.0(\mathrm{Cq}), 144.9(\mathrm{Cq}), 137.3(\mathrm{Cq}), 136.0(\mathrm{Cq}), 134.4\left(\mathrm{CH}_{\mathrm{Ar}}\right), 131.9\left(\mathrm{CH}_{\mathrm{Ar}}\right), 130.2(\mathrm{Cq}), 129.7$ $\left(\mathrm{CH}_{\mathrm{Ar}}\right), 128.0\left(\mathrm{CH}_{\mathrm{Ar}}\right), 127.9\left(\mathrm{CH}_{\mathrm{Ar}}\right), 124.5\left(\mathrm{CH}_{\mathrm{Ar}}\right), 111.6\left(\mathrm{CH}_{2}\right), 78.3(\mathrm{Cq}), 48.8(\mathrm{CH}), 47.8\left(\mathrm{CH}_{2}\right), 32.4$ $\left(\mathrm{CH}_{2}\right), 32.2\left(\mathrm{CH}_{2}\right), 30.9\left(\mathrm{CH}_{2}\right), 25.6\left(\mathrm{CH}_{2}\right), 24.6\left(\mathrm{CH}_{2}\right)$. HRMS (EI) $\mathrm{m} / \mathrm{z}$ : calculated for $\mathrm{C}_{26} \mathrm{H}_{27} \mathrm{CIN}_{3} \mathrm{O}_{5}$ $\left[\mathrm{M}+\mathrm{H}^{+}\right]$496.1634; found 496.1642 .

General procedure for the synthesis of pyrrolobenzodiazepine-5-ones 12a-r. The corresponding pyrrolo derivative 6,7 or $11(1.0 \mathrm{mmol}, 1.0$ equiv., $0.1 \mathrm{M})$ was dissolved in ethanol (10 mL) and $\mathrm{SnCl}_{2} \cdot 2 \mathrm{H}_{2} \mathrm{O}(10 \mathrm{mmol}, 10$ equiv.) and $\mathrm{HCl} 1 \mathrm{M}(3.0 \mathrm{mmol}, 3.0$ equiv.) were succesively added to the solution. The mixture was stirred at $70^{\circ} \mathrm{C}$ for one hour, after which the solvent was removed in a rotary evaporator. The raw product was dissolved in chloroform and washed with water (in each washing a saturated solution of $\mathrm{Na}_{2} \mathrm{CO}_{3}$ was added to the aqueous phase). The organic phase was dried over anhydrous sodium sulfate, filtered and concentrated to dryness, thus yielding the corresponding pyrrolobenzodiazepine-5-ones 12a-r.

\section{$N$-Cyclohexyl-5-oxo-11-phenyl-2,3,5,11a-tetrahydro-1H-benzo[e]pyrrolo[1,2-}

a][1,4]diazepine-11a-carboxamide (12a). Light brown solid. Yield: $321 \mathrm{mg}, 80 \%$. M. p. 78-80 ${ }^{\circ} \mathrm{C} .{ }^{1} \mathrm{H}$ NMR $\left(300 \mathrm{MHz}, \mathrm{CDCl}_{3}\right)$ 8: $7.94(\mathrm{~d}, J=7.9 \mathrm{~Hz}, 1 \mathrm{H}), 7.57-7.40(\mathrm{~m}, 6 \mathrm{H}), 7.32-7.24(\mathrm{~m}, 2 \mathrm{H})$, $5.52(\mathrm{~d}, J=8.6 \mathrm{~Hz}, 1 \mathrm{H}, \mathrm{NH}), 3.98-3.81(\mathrm{~m}, 2 \mathrm{H}), 3.39-3.27(\mathrm{~m}, 1 \mathrm{H}), 2.68(\mathrm{dt}, J=12.4,6.0 \mathrm{~Hz}, 1 \mathrm{H})$, $2.25(\mathrm{ddd}, J=13.7,8.8,6.0 \mathrm{~Hz}, 1 \mathrm{H}), 1.76(\mathrm{tt}, J=12.4,6.0 \mathrm{~Hz}, 1 \mathrm{H}), 1.66-0.41(\mathrm{~m}, 11 \mathrm{H}) .{ }^{13} \mathrm{C} \mathrm{NMR}$ \{DEPT-135\} (75 MHz, CDCl 3 ) ס: $171.1(\mathrm{Cq}), 169.1(\mathrm{Cq}), 166.0(\mathrm{Cq}), 145.1(\mathrm{Cq}), 138.9(\mathrm{Cq}), 132.1$ 
$\left(\mathrm{CH}_{\mathrm{Ar}}\right), 129.6\left(\mathrm{CH}_{\mathrm{Ar}}\right), 129.1\left(\mathrm{CH}_{\mathrm{Ar}}\right), 128.2\left(\mathrm{CH}_{\mathrm{Ar}}\right), 127.7\left(\mathrm{CH}_{\mathrm{Ar}}\right), 127.3\left(\mathrm{CH}_{\mathrm{Ar}}\right), 126.4\left(\mathrm{CH}_{\mathrm{Ar}}\right), 125.0$ (Cq), $70.1(\mathrm{Cq}), 49.0\left(\mathrm{CH}_{2}\right), 48.4(\mathrm{CH}), 40.2\left(\mathrm{CH}_{2}\right), 32.5\left(\mathrm{CH}_{2}\right), 32.2\left(\mathrm{CH}_{2}\right), 25.2\left(\mathrm{CH}_{2}\right), 24.9\left(\mathrm{CH}_{2}\right)$, $24.7\left(\mathrm{CH}_{2}\right), 22.3\left(\mathrm{CH}_{2}\right)$. HRMS (ESI-QTOF) $\mathrm{m} / \mathrm{z}$ : calculated for $\mathrm{C}_{25} \mathrm{H}_{28} \mathrm{~N}_{3} \mathrm{O}_{2}\left[\mathrm{M}+\mathrm{H}^{+}\right]$402.2176; found 402.2179 .

\section{$N$-Cyclohexyl-5-oxo-11-(p-tolyl)-2,3,5,11a-tetrahydro-1H-benzo[e]pyrrolo[1,2-}

a][1,4]diazepine-11a-carboxamide (12b). Light brown solid. Yield: 332 mg, 80\%. M. p. 120-121 ${ }^{\circ} \mathrm{C} .{ }^{1} \mathrm{H}$ NMR $\left(300 \mathrm{MHz}, \mathrm{CDCl}_{3}\right)$ 8: $7.96(\mathrm{dd}, J=7.8,1.5 \mathrm{~Hz}, 1 \mathrm{H}), 7.52-7.44(\mathrm{~m}, 3 \mathrm{H}), 7.35-7.23(\mathrm{~m}$, $4 \mathrm{H}), 5.37(\mathrm{~d}, J=8.6 \mathrm{~Hz}, 1 \mathrm{H}, \mathrm{NH}), 3.99-3.79(\mathrm{~m}, 2 \mathrm{H}), 3.42-3.29(\mathrm{~m}, 1 \mathrm{H}), 2.74(\mathrm{dt}, J=12.8,6.1 \mathrm{~Hz}$, $1 \mathrm{H}), 2.40(\mathrm{~s}, 3 \mathrm{H}), 2.32-2.22(\mathrm{~m}, 2 \mathrm{H}), 1.82-0.81(\mathrm{~m}, 10 \mathrm{H}), 0.52-0.39(\mathrm{~m}, 1 \mathrm{H}) .{ }^{13} \mathrm{C}$ NMR $\{\mathrm{DEPT}-135\}$ $\left(75 \mathrm{MHz}, \mathrm{CDCl}_{3}\right)$ \&: $171.1(\mathrm{Cq}), 169.2(\mathrm{Cq}), 166.0(\mathrm{Cq}), 145.1(\mathrm{Cq}), 139.2(\mathrm{Cq}), 136.1(\mathrm{Cq}), 132.0$ $\left(\mathrm{CH}_{\mathrm{Ar}}\right), 129.5\left(\mathrm{CH}_{\mathrm{Ar}}\right), 128.8\left(\mathrm{CH}_{\mathrm{Ar}}\right), 127.7\left(\mathrm{CH}_{\mathrm{Ar}}\right), 127.3\left(\mathrm{CH}_{\mathrm{Ar}}\right), 126.3\left(\mathrm{CH}_{\mathrm{Ar}}\right), 125.0(\mathrm{Cq}), 70.1(\mathrm{Cq})$, $48.9\left(\mathrm{CH}_{2}\right), 48.3(\mathrm{CH}), 40.3\left(\mathrm{CH}_{2}\right), 32.5\left(\mathrm{CH}_{2}\right), 32.2\left(\mathrm{CH}_{2}\right), 25.2\left(\mathrm{CH}_{2}\right), 24.9\left(\mathrm{CH}_{2}\right), 24.7\left(\mathrm{CH}_{2}\right), 22.3$ $\left(\mathrm{CH}_{2}\right), 21.4\left(\mathrm{CH}_{3}\right)$. HRMS (ESI-QTOF) $\mathrm{m} / \mathrm{z}$ : calculated for $\mathrm{C}_{26} \mathrm{H}_{30} \mathrm{~N}_{3} \mathrm{O}_{2}\left[\mathrm{M}+\mathrm{H}^{+}\right]$416.2333; found 416.2335.

\section{1-(4-Chlorophenyl)- $N$-cyclohexyl-5-oxo-2,3,5,11a-tetrahydro-1H-benzo[e]pyrrolo[1,2-}

a][1,4]diazepine-11a-carboxamide (12c). Light brown solid. Yield: 349 mg, 80\%. M. p. 178-180 ${ }^{\circ} \mathrm{C} .{ }^{1} \mathrm{H}$ NMR $\left(300 \mathrm{MHz}, \mathrm{CDCl}_{3}\right)$ 8: 7.97-7.94 (m, 1H), 7.55-7.23 (m, 7H), $5.43(\mathrm{~d}, J=8.4 \mathrm{~Hz}, 1 \mathrm{H}$, $\mathrm{NH}), 3.99-3.80(\mathrm{~m}, 2 \mathrm{H}), 3.40-3.28(\mathrm{~m}, 1 \mathrm{H}), 2.64(\mathrm{dt}, J=13.1,6.4 \mathrm{~Hz}, 1 \mathrm{H}), 2.28(\mathrm{ddd}, J=13.9,8.4$, $6.4 \mathrm{~Hz}, 1 \mathrm{H}), 1.84-1.73(\mathrm{~m}, 2 \mathrm{H}), 1.64-0.31(\mathrm{~m}, 10 \mathrm{H}) .{ }^{13} \mathrm{C}$ NMR $\left\{\right.$ DEPT-135\} $\left(75 \mathrm{MHz}, \mathrm{CDCl}_{3}\right) \delta:$ $170.1(\mathrm{Cq}), 169.0(\mathrm{Cq}), 165.8(\mathrm{Cq}), 144.8(\mathrm{Cq}), 137.4(\mathrm{Cq}), 135.2(\mathrm{Cq}), 132.1\left(\mathrm{CH}_{\mathrm{Ar}}\right), 129.5\left(\mathrm{CH}_{\mathrm{Ar}}\right)$, $129.2\left(\mathrm{CH}_{\mathrm{Ar}}\right), 128.5\left(\mathrm{CH}_{\mathrm{Ar}}\right), 127.3\left(\mathrm{CH}_{\mathrm{Ar}}\right), 126.6\left(\mathrm{CH}_{\mathrm{Ar}}\right), 124.9(\mathrm{Cq}), 70.0(\mathrm{Cq}), 49.0\left(\mathrm{CH}_{2}\right), 48.4$ (CH), $40.3\left(\mathrm{CH}_{2}\right), 32.5\left(\mathrm{CH}_{2}\right), 32.2\left(\mathrm{CH}_{2}\right), 25.1\left(\mathrm{CH}_{2}\right), 24.9\left(\mathrm{CH}_{2}\right), 24.6\left(\mathrm{CH}_{2}\right), 22.3\left(\mathrm{CH}_{2}\right)$. HRMS (ESI-QTOF) $\mathrm{m} / \mathrm{z}$ : calculated for $\mathrm{C}_{25} \mathrm{H}_{27} \mathrm{CIN}_{3} \mathrm{O}_{2}\left[\mathrm{M}+\mathrm{H}^{+}\right]$436.1786; found 436.1788 .

$N$-(tert-Butyl)-5-oxo-11-phenyl-2,3,5,11a-tetrahydro-1H-benzo[e]pyrrolo[1,2a][1,4]diazepine-11a-carboxamide (12d). Light brown solid. Yield: 353 mg, 94\%. M. p. 105-106 
${ }^{\circ} \mathrm{C} .{ }^{1} \mathrm{H}$ NMR $\left(300 \mathrm{MHz}, \mathrm{CDCl}_{3}\right)$ 8: 7.99-7.96 (m, 1H), 7.58-7.25 (m, 8H), $5.44(\mathrm{~s}, 1 \mathrm{H}, \mathrm{NH}), 4.02-$ $3.93(\mathrm{~m}, 1 \mathrm{H}), 3.87-3.77(\mathrm{~m}, 1 \mathrm{H}), 2.72(\mathrm{dt}, J=11.8,5.8 \mathrm{~Hz}, 1 \mathrm{H}), 2.31-2.21(\mathrm{~m}, 1 \mathrm{H}), 1.77(\mathrm{tt}, J=$ 11.8, $5.8 \mathrm{~Hz}, 1 \mathrm{H}), 1.64-1.53(\mathrm{~m}, 1 \mathrm{H}), 0.91(\mathrm{~s}, 9 \mathrm{H}) .{ }^{13} \mathrm{C}$ NMR $\left\{\right.$ DEPT-135\} $\left(75 \mathrm{MHz}, \mathrm{CDCl}_{3}\right) \delta: 171.4$ $(\mathrm{Cq}), 169.2(\mathrm{Cq}), 166.2(\mathrm{Cq}), 145.2(\mathrm{Cq}), 138.8(\mathrm{Cq}), 132.0\left(\mathrm{CH}_{\mathrm{Ar}}\right), 129.3\left(\mathrm{CH}_{\mathrm{Ar}}\right), 129.1\left(\mathrm{CH}_{\mathrm{Ar}}\right)$, 128.2 $\left(\mathrm{CH}_{\mathrm{Ar}}\right), 127.7\left(\mathrm{CH}_{\mathrm{Ar}}\right), 127.4\left(\mathrm{CH}_{\mathrm{Ar}}\right), 126.4\left(\mathrm{CH}_{\mathrm{Ar}}\right), 125.3(\mathrm{Cq}), 70.6(\mathrm{Cq}), 51.4(\mathrm{Cq}), 48.9\left(\mathrm{CH}_{2}\right)$, $39.9\left(\mathrm{CH}_{2}\right), 27.9\left(\mathrm{CH}_{3}\right), 22.3\left(\mathrm{CH}_{2}\right)$. HRMS (ESI-QTOF) $\mathrm{m} / \mathrm{z}$ : calculated for $\mathrm{C}_{23} \mathrm{H}_{26} \mathrm{~N}_{3} \mathrm{O}_{2}\left[\mathrm{M}+\mathrm{H}^{+}\right]$ 376.2020; found 376.2022.

$N$-Butyl-5-oxo-11-phenyl-2,3,5,11a-tetrahydro-1H-benzo[e]pyrrolo[1,2-a][1,4]diazepine-11acarboxamide (12e). Light brown solid. Yield: $357 \mathrm{mg}, 95 \%$. M. p. $149-150^{\circ} \mathrm{C} .{ }^{1} \mathrm{H}$ NMR $(300 \mathrm{MHz}$, $\left.\mathrm{CDCl}_{3}\right) \delta: 7.92(\mathrm{dd}, J=8.2,1.2 \mathrm{~Hz}, 1 \mathrm{H}), 7.58-7.40(\mathrm{~m}, 6 \mathrm{H}), 7.32(\mathrm{dd}, J=8.2,1.2 \mathrm{~Hz}, 1 \mathrm{H}), 7.28-$ $7.23(\mathrm{~m}, 1 \mathrm{H}), 5.98(\mathrm{t}, J=5.8 \mathrm{~Hz}, 1 \mathrm{H}, \mathrm{NH}), 3.97-3.81(\mathrm{~m}, 2 \mathrm{H}), 3.02-2.90(\mathrm{~m}, 1 \mathrm{H}), 2.77-2.63(\mathrm{~m}$, $2 \mathrm{H}), 2.26(\mathrm{ddd}, J=13.5,8.7,6.5 \mathrm{~Hz}, 1 \mathrm{H}), 1.83-1.71(\mathrm{~m}, 1 \mathrm{H}), 1.66-1.52(\mathrm{~m}, 1 \mathrm{H}), 1.02-0.80(\mathrm{~m}$, 4H), $0.71(\mathrm{t}, J=6.9 \mathrm{~Hz}, 3 \mathrm{H}) .{ }^{13} \mathrm{C}$ NMR $\{\mathrm{DEPT}-135\}\left(75 \mathrm{MHz}, \mathrm{CDCl}_{3}\right) \delta: 171.0(\mathrm{Cq}), 170.1$ (Cq), $165.9(\mathrm{Cq}), 145.0(\mathrm{Cq}), 139.0(\mathrm{Cq}), 132.0\left(\mathrm{CH}_{\mathrm{Ar}}\right), 129.5\left(\mathrm{CH}_{\mathrm{Ar}}\right), 129.1\left(\mathrm{CH}_{\mathrm{Ar}}\right), 128.2\left(\mathrm{CH}_{\mathrm{Ar}}\right), 127.7$ $\left(\mathrm{CH}_{\mathrm{Ar}}\right), 127.4\left(\mathrm{CH}_{\mathrm{Ar}}\right), 126.3\left(\mathrm{CH}_{\mathrm{Ar}}\right), 124.9(\mathrm{Cq}), 70.1(\mathrm{Cq}), 49.0\left(\mathrm{CH}_{2}\right), 40.2\left(\mathrm{CH}_{2}\right), 39.4\left(\mathrm{CH}_{2}\right), 31.3$ $\left(\mathrm{CH}_{2}\right), 22.3\left(\mathrm{CH}_{2}\right), 19.9\left(\mathrm{CH}_{2}\right), 13.7\left(\mathrm{CH}_{3}\right)$. HRMS (ESI-QTOF) $\mathrm{m} / \mathrm{z}$ : calculated for $\mathrm{C}_{23} \mathrm{H}_{26} \mathrm{~N}_{3} \mathrm{O}_{2}$ $\left[\mathrm{M}+\mathrm{H}^{+}\right]$376.2020; found 376.2016.

\section{7-Chloro-N-cyclohexyl-5-oxo-11-phenyl-2,3,5,11a-tetrahydro-1H-benzo[e]pyrrolo[1,2-}

a][1,4]diazepine-11a-carboxamide (12f). Light brown solid. Yield: 305 mg, 70\%. M. p. 178-179 ${ }^{\circ} \mathrm{C} .{ }^{1} \mathrm{H}$ NMR $\left(300 \mathrm{MHz}, \mathrm{CDCl}_{3}\right)$ 8: $7.91(\mathrm{~d}, J=2.3 \mathrm{~Hz}, 1 \mathrm{H}), 7.54-7.40(\mathrm{~m}, 6 \mathrm{H}), 7.25(\mathrm{~d}, J=8.6 \mathrm{~Hz}$, $1 \mathrm{H}), 5.63(\mathrm{~d}, J=8.6 \mathrm{~Hz}, 1 \mathrm{H}, \mathrm{NH}), 3.96-3.80(\mathrm{~m}, 2 \mathrm{H}), 3.44-3.32(\mathrm{~m}, 1 \mathrm{H}), 2.69(\mathrm{dt}, J=12.7,6.4 \mathrm{~Hz}$, $1 \mathrm{H}), 2.26(\mathrm{ddd}, J=14.1,8.6,6.4 \mathrm{~Hz}, 1 \mathrm{H}), 1.84-1.48(\mathrm{~m}, 6 \mathrm{H}), 1.22-0.51(\mathrm{~m}, 6 \mathrm{H}) .{ }^{13} \mathrm{C}$ NMR \{DEPT$135\}\left(75 \mathrm{MHz}, \mathrm{CDCl}_{3}\right)$ 8: $171.6(\mathrm{Cq}), 168.9(\mathrm{Cq}), 164.7(\mathrm{Cq}), 143.6(\mathrm{Cq}), 138.7(\mathrm{Cq}), 132.1\left(\mathrm{CH}_{\mathrm{Ar}}\right)$, $129.3\left(\mathrm{CH}_{\mathrm{Ar}}\right), 129.1\left(\mathrm{CH}_{\mathrm{Ar}}\right), 129.0\left(\mathrm{CH}_{\mathrm{Ar}}\right), 128.3\left(\mathrm{CH}_{\mathrm{Ar}}\right), 127.6\left(\mathrm{CH}_{\mathrm{Ar}}\right), 126.4(\mathrm{Cq}), 70.2(\mathrm{Cq}), 49.0$ $\left(\mathrm{CH}_{2}\right), 48.5(\mathrm{CH}), 40.0\left(\mathrm{CH}_{2}\right), 32.5\left(\mathrm{CH}_{2}\right), 32.3\left(\mathrm{CH}_{2}\right), 25.2\left(\mathrm{CH}_{2}\right), 24.9\left(\mathrm{CH}_{2}\right), 24.7\left(\mathrm{CH}_{2}\right), 22.3\left(\mathrm{CH}_{2}\right)$. HRMS (ESI-QTOF) $m / z$ : calculated for $\mathrm{C}_{25} \mathrm{H}_{27} \mathrm{ClN}_{3} \mathrm{O}_{2}\left[\mathrm{M}+\mathrm{H}^{+}\right]$436.1786; found 436.1783 . 


\section{8-Chloro- $N$-cyclohexyl-5-oxo-11-( $p$-tolyl)-2,3,5,11a-tetrahydro-1H-benzo[e]pyrrolo[1,2-}

a][1,4]diazepine-11a-carboxamide (12g). Light brown solid. Yield: 351 mg, 78\%. M. p. 158-160 ${ }^{\circ} \mathrm{C} .{ }^{1} \mathrm{H}$ NMR $\left(300 \mathrm{MHz}, \mathrm{CDCl}_{3}\right)$ 8: $7.90(\mathrm{~d}, J=8.5 \mathrm{~Hz}, 1 \mathrm{H}), 7.44(\mathrm{~d}, J=8.2 \mathrm{~Hz}, 2 \mathrm{H}), 7.35(\mathrm{~d}, J=2.1$ $\mathrm{Hz}, 1 \mathrm{H}), 7.27-7.24(\mathrm{~m}, 3 \mathrm{H}), 5.53(\mathrm{~d}, J=8.5 \mathrm{~Hz}, 1 \mathrm{H}, \mathrm{NH}), 3.96-3.81(\mathrm{~m}, 2 \mathrm{H}), 3.48-3.34(\mathrm{~m}, 1 \mathrm{H})$, $2.76(\mathrm{dt}, J=13.0,6.0 \mathrm{~Hz}, 1 \mathrm{H}), 2.41(\mathrm{~s}, 3 \mathrm{H}), 2.33-2.23(\mathrm{~m}, 1 \mathrm{H}), 1.85-0.84(\mathrm{~m}, 11 \mathrm{H}), 0.61-0.48(\mathrm{~m}$, 1H). ${ }^{13} \mathrm{C}$ NMR $\{$ DEPT-135\} (75 MHz, CDCl $)$ 8: 172.7 (Cq), 168.9 (Cq), 165.4 (Cq), 146.1 (Cq), $139.5(\mathrm{Cq}), 137.9(\mathrm{Cq}), 135.7(\mathrm{Cq}), 131.0\left(\mathrm{CH}_{\mathrm{Ar}}\right), 128.9\left(\mathrm{CH}_{\mathrm{Ar}}\right), 127.6\left(\mathrm{CH}_{\mathrm{Ar}}\right), 127.1\left(\mathrm{CH}_{\mathrm{Ar}}\right), 126.5$ $\left(\mathrm{CH}_{\mathrm{Ar}}\right), 123.5(\mathrm{Cq}), 70.3(\mathrm{Cq}), 49.1\left(\mathrm{CH}_{2}\right), 48.5(\mathrm{CH}), 40.1\left(\mathrm{CH}_{2}\right), 32.5\left(\mathrm{CH}_{2}\right), 32.3\left(\mathrm{CH}_{2}\right), 25.2\left(\mathrm{CH}_{2}\right)$, $24.9\left(\mathrm{CH}_{2}\right), 24.7\left(\mathrm{CH}_{2}\right), 22.3\left(\mathrm{CH}_{2}\right), 21.4\left(\mathrm{CH}_{3}\right)$. HRMS (ESI-QTOF) $\mathrm{m} / \mathrm{z}$ : calculated for $\mathrm{C}_{26} \mathrm{H}_{28} \mathrm{ClNaN}_{3} \mathrm{O}_{2}\left[\mathrm{M}+\mathrm{Na}^{+}\right]$472.1762; found 472.1763.

\section{$N$-(tert-Butyl)-8-chloro-5-oxo-11-phenyl-2,3,5,11a-tetrahydro-1H-benzo[e]pyrrolo[1,2-}

a][1,4]diazepine-11a-carboxamide (12h). Light brown solid. Yield: 311 mg, 76\%. M. p. 215-216 ${ }^{\circ} \mathrm{C} .{ }^{1} \mathrm{H}$ NMR $\left(300 \mathrm{MHz}, \mathrm{CDCl}_{3}\right)$ 8: $7.89(\mathrm{~d}, J=8.5 \mathrm{~Hz}, 1 \mathrm{H}), 7.52-7.23(\mathrm{~m}, 7 \mathrm{H}), 5.30(\mathrm{~s}, 1 \mathrm{H}, \mathrm{NH})$, 3.95-3.75 (m, 2H), $2.69(\mathrm{dt}, J=13.5,5.7 \mathrm{~Hz}, 1 \mathrm{H}), 2.24(\mathrm{ddd}, J=13.5,9.1,6.6 \mathrm{~Hz}, 1 \mathrm{H}), 1.81-1.69$ (m, 1H), 1.65-1.49 (m, 1H), $0.93(\mathrm{~s}, 9 \mathrm{H}) .{ }^{13} \mathrm{C}$ NMR $\{\mathrm{DEPT}-135\}\left(75 \mathrm{MHz}, \mathrm{CDCl}_{3}\right) \delta: 172.7$ (Cq), $168.9(\mathrm{Cq}), 165.1(\mathrm{Cq}), 146.1(\mathrm{Cq}), 138.4(\mathrm{Cq}), 137.8(\mathrm{Cq}), 130.9\left(\mathrm{CH}_{\text {Ar }}\right), 129.3\left(\mathrm{CH}_{\text {Ar }}\right), 128.2$ $\left(\mathrm{CH}_{\mathrm{Ar}}\right), 127.6\left(\mathrm{CH}_{\mathrm{Ar}}\right), 126.9\left(\mathrm{CH}_{\mathrm{Ar}}\right), 126.6\left(\mathrm{CH}_{\mathrm{Ar}}\right), 123.8(\mathrm{Cq}), 70.7(\mathrm{Cq}), 51.6(\mathrm{Cq}), 49.0\left(\mathrm{CH}_{2}\right), 39.8$ $\left(\mathrm{CH}_{2}\right), 27.9\left(\mathrm{CH}_{3}\right), 22.3\left(\mathrm{CH}_{2}\right)$. HRMS (ESI-QTOF) $\mathrm{m} / \mathrm{z}$ : calculated for $\mathrm{C}_{23} \mathrm{H}_{25} \mathrm{ClN}_{3} \mathrm{O}_{2}\left[\mathrm{M}+\mathrm{H}^{+}\right]$ 410.1630; found 410.1629 .

\section{8-Chloro-N-cyclohexyl-11-(4-methoxyphenyl)-5-oxo-2,3,5,11a-tetrahydro-1H-}

benzo[e]pyrrolo[1,2-a][1,4]diazepine-11a-carboxamide (12i). Light brown solid. Yield: $382 \mathrm{mg}$, 82\%. M. p. $114-115{ }^{\circ} \mathrm{C} .{ }^{1} \mathrm{H} N M R\left(300 \mathrm{MHz}, \mathrm{CDCl}_{3}\right)$ 8: $7.90(\mathrm{~d}, J=8.5 \mathrm{~Hz}, 1 \mathrm{H}), 7.49(\mathrm{~d}, J=8.8 \mathrm{~Hz}$, 2H), $7.34(\mathrm{~d}, J=2.1 \mathrm{~Hz}, 1 \mathrm{H}), 7.25(\mathrm{dd}, J=8.5,2.1 \mathrm{~Hz}, 1 \mathrm{H}), 6.96(\mathrm{~d}, J=8.8 \mathrm{~Hz}, 2 \mathrm{H}), 5.32(\mathrm{~d}, J=8.2$ $\mathrm{Hz}, 1 \mathrm{H}, \mathrm{NH}), 3.85(\mathrm{~s}, 3 \mathrm{H}), 3.89-3.83(\mathrm{~m}, 2 \mathrm{H}), 3.47-3.35(\mathrm{~m}, 1 \mathrm{H}), 2.78(\mathrm{dt}, J=13.5,6.4 \mathrm{~Hz}, 1 \mathrm{H})$, $2.30(\mathrm{ddd}, J=13.5,8.3,6.4 \mathrm{~Hz}, 1 \mathrm{H}), 1.86-1.48(\mathrm{~m}, 6 \mathrm{H}), 1.27-0.47(\mathrm{~m}, 6 \mathrm{H}) .{ }^{13} \mathrm{C}$ NMR $\{$ DEPT- 135$\}$ $\left(75 \mathrm{MHz}, \mathrm{CDCl}_{3}\right)$ 8: $171.8(\mathrm{Cq}), 168.8(\mathrm{Cq}), 165.3(\mathrm{Cq}), 160.5(\mathrm{Cq}), 146.1(\mathrm{Cq}), 137.8(\mathrm{Cq}), 131.1$ 
$\left(\mathrm{CH}_{\mathrm{Ar}}\right), 129.4\left(\mathrm{CH}_{\mathrm{Ar}}\right), 127.0\left(\mathrm{CH}_{\mathrm{Ar}}\right), 126.4\left(\mathrm{CH}_{\mathrm{Ar}}\right), 123.4(\mathrm{Cq}), 113.6\left(\mathrm{CH}_{\mathrm{Ar}}\right), 70.4(\mathrm{Cq}), 55.4\left(\mathrm{CH}_{3}\right)$, $49.1\left(\mathrm{CH}_{2}\right), 48.5(\mathrm{CH}), 40.3\left(\mathrm{CH}_{2}\right), 32.5\left(\mathrm{CH}_{2}\right), 32.3\left(\mathrm{CH}_{2}\right), 25.1\left(\mathrm{CH}_{2}\right), 24.9\left(\mathrm{CH}_{2}\right), 24.7\left(\mathrm{CH}_{2}\right), 22.3$ ( $\left.\mathrm{CH}_{2}\right)$. HRMS (ESI-QTOF) $\mathrm{m} / \mathrm{z}$ : calculated for $\mathrm{C}_{26} \mathrm{H}_{29} \mathrm{CIN}_{3} \mathrm{O}_{3}\left[\mathrm{M}+\mathrm{H}^{+}\right]$466.1892; found 466.1890 . N-Cyclohexyl-5-oxo-11-phenyl-5,11a-dihydro-3H-benzo[e]pyrrolo[1,2-a][1,4]diazepine-11acarboxamide (12j). Brown solid. Yield: $379 \mathrm{mg}, 95 \%$. M. p. $70-72{ }^{\circ} \mathrm{C} .{ }^{1} \mathrm{H} \mathrm{NMR}\left(300 \mathrm{MHz}, \mathrm{CDCl}_{3}\right)$

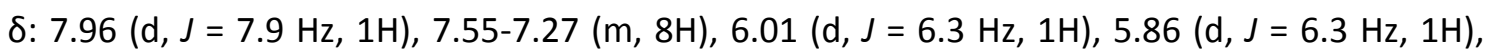
$5.44(\mathrm{~d}, J=8.5 \mathrm{~Hz}, 1 \mathrm{H}, \mathrm{NH}), 4.73-4.51(\mathrm{~m}, 2 \mathrm{H}), 3.36-3.23(\mathrm{~m}, 1 \mathrm{H}), 1.58-0.37(\mathrm{~m}, 10 \mathrm{H}) .{ }^{13} \mathrm{C} \mathrm{NMR}$ \{DEPT-135\} (75 MHz, CDCl $)$ ) ס: $169.4(\mathrm{Cq}), 166.8(\mathrm{Cq}), 165.4(\mathrm{Cq}), 145.2(\mathrm{Cq}), 139.2(\mathrm{Cq}), 132.2$ (CH), $130.5(\mathrm{CH}), 129.5(\mathrm{CH}), 129.2(\mathrm{CH}), 128.2(\mathrm{CH}), 127.8(\mathrm{CH}), 127.2(\mathrm{CH}), 127.0(\mathrm{CH}), 126.3$ (CH), $124.7(\mathrm{Cq}), 76.2(\mathrm{Cq}), 55.2\left(\mathrm{CH}_{2}\right), 48.4(\mathrm{CH}), 32.3\left(\mathrm{CH}_{2}\right), 32.2\left(\mathrm{CH}_{2}\right), 25.1\left(\mathrm{CH}_{2}\right), 24.8\left(\mathrm{CH}_{2}\right)$, 24.6 $\left(\mathrm{CH}_{2}\right)$. HRMS (ESI-QTOF) $\mathrm{m} / \mathrm{z}$ : calculated for $\mathrm{C}_{25} \mathrm{H}_{26} \mathrm{~N}_{3} \mathrm{O}_{2}\left[\mathrm{M}+\mathrm{H}^{+}\right]$400.2020; found 400.2024. $N$-Cyclohexyl-5-oxo-11-(p-tolyl)-5,11a-dihydro-3H-benzo[e]pyrrolo[1,2-a][1,4]diazepine-11acarboxamide (12k). Brown solid. Yield: $347 \mathrm{mg}, 84 \%$. M. p. $168-170{ }^{\circ} \mathrm{C} .{ }^{1} \mathrm{H}$ NMR $(300 \mathrm{MHz}$,

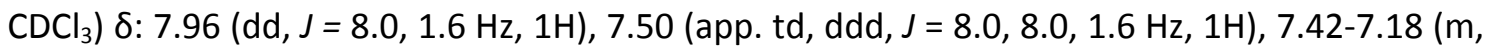
$6 \mathrm{H}), 6.00(\mathrm{dt}, J=6.2,2.0 \mathrm{~Hz}, 1 \mathrm{H}), 5.89(\mathrm{dt}, J=6.2,2.0 \mathrm{~Hz}, 1 \mathrm{H}), 5.42(\mathrm{~d}, J=8.5 \mathrm{~Hz}, 1 \mathrm{H}, \mathrm{NH}), 4.65$ (dt, $J=17.5,2.1 \mathrm{~Hz}, 1 \mathrm{H}), 4.56(\mathrm{dt}, J=17.5,2.1 \mathrm{~Hz}, 1 \mathrm{H}), 3.34-3.25(\mathrm{~m}, 1 \mathrm{H}), 2.36(\mathrm{~s}, 3 \mathrm{H}), 1.68-0.39$

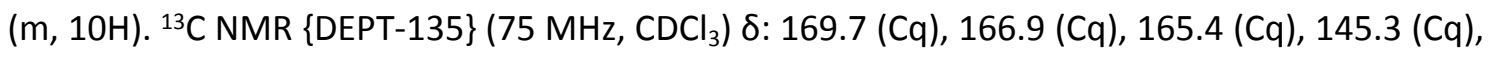
$139.4(\mathrm{Cq}), 136.4(\mathrm{Cq}), 132.2(\mathrm{CH}), 130.6(\mathrm{CH}), 129.5(\mathrm{CH}), 128.8(\mathrm{CH}), 127.9(\mathrm{CH}), 127.2(\mathrm{CH})$, $126.9(\mathrm{CH}), 126.2(\mathrm{CH}), 124.7(\mathrm{Cq}), 76.2(\mathrm{Cq}), 55.2\left(\mathrm{CH}_{2}\right), 48.4(\mathrm{CH}), 32.3\left(\mathrm{CH}_{2}\right), 32.2\left(\mathrm{CH}_{2}\right), 25.1$ $\left(\mathrm{CH}_{2}\right), 24.8\left(\mathrm{CH}_{2}\right), 24.6\left(\mathrm{CH}_{2}\right), 21.4\left(\mathrm{CH}_{3}\right)$. HRMS (ESI-QTOF) $\mathrm{m} / \mathrm{z}$ : calculated for $\mathrm{C}_{26} \mathrm{H}_{28} \mathrm{~N}_{3} \mathrm{O}_{2}$ $\left[\mathrm{M}+\mathrm{H}^{+}\right]$414.2176; found 414.2181.

\section{N-Cyclohexyl-11-(4-methoxyphenyl)-5-oxo-5,11a-dihydro-3H-benzo[e]pyrrolo[1,2-}

a][1,4]diazepine-11a-carboxamide (12I). Brown solid. Yield: $292 \mathrm{mg}, 68 \%$. M. p. $178-180^{\circ} \mathrm{C} .{ }^{1} \mathrm{H}$ NMR (300 MHz, $\left.\mathrm{CDCl}_{3}\right)$ ): $7.95(\mathrm{dd}, J=8.0,1.4 \mathrm{~Hz}, 1 \mathrm{H}), 7.53-7.45(\mathrm{~m}, 3 \mathrm{H}), 7.36(\mathrm{dd}, J=8.0,1.4$ $\mathrm{Hz}, 1 \mathrm{H}$ ), 7.27 (app. td, ddd, $J=8.0,8.0,1.4 \mathrm{~Hz}, 1 \mathrm{H}), 6.90(\mathrm{~d}, J=8.8 \mathrm{~Hz}, 2 \mathrm{H}$ ), 6.03 (dt, $J=6.5,2.0$ 
$\mathrm{Hz}, 1 \mathrm{H}), 5.93(\mathrm{dt}, J=6.5,2.0 \mathrm{~Hz}, 1 \mathrm{H}), 5.40(\mathrm{~d}, J=8.5 \mathrm{~Hz}, 1 \mathrm{H}, \mathrm{NH}), 4.66(\mathrm{dt}, J=17.5,2.1 \mathrm{~Hz}, 1 \mathrm{H})$, $4.56(\mathrm{dt}, J=17.5,2.1 \mathrm{~Hz}, 1 \mathrm{H}), 3.81(\mathrm{~s}, 3 \mathrm{H}), 3.33-3.23(\mathrm{~m}, 1 \mathrm{H}), 1.55-0.36(\mathrm{~m}, 10 \mathrm{H}) .{ }^{13} \mathrm{C} \mathrm{NMR}$ \{DEPT-135\} (75 MHz, CDCl 3 ) ס: $168.7(\mathrm{Cq}), 166.8(\mathrm{Cq}), 165.4(\mathrm{Cq}), 160.5(\mathrm{Cq}), 145.3(\mathrm{Cq}), 132.2$ (CH), $131.7(\mathrm{Cq}), 130.6(\mathrm{CH}), 129.8(\mathrm{CH}), 129.5(\mathrm{CH}), 127.2(\mathrm{CH}), 126.9(\mathrm{CH}), 126.1(\mathrm{CH}), 124.6$ (Cq), $113.5(\mathrm{CH}), 76.4(\mathrm{Cq}), 55.3\left(\mathrm{CH}_{3}\right), 55.2\left(\mathrm{CH}_{2}\right), 48.4(\mathrm{CH}), 32.3\left(\mathrm{CH}_{2}\right), 32.2\left(\mathrm{CH}_{2}\right), 25.1\left(\mathrm{CH}_{2}\right)$, $24.8\left(\mathrm{CH}_{2}\right), 24.6\left(\mathrm{CH}_{2}\right)$. HRMS (ESI-QTOF) $\mathrm{m} / \mathrm{z}$ : calculated for $\mathrm{C}_{26} \mathrm{H}_{28} \mathrm{~N}_{3} \mathrm{O}_{3}\left[\mathrm{M}+\mathrm{H}^{+}\right]$430.2125; found 430.2132 .

$N$-(tert-Butyl)-5-oxo-11-phenyl-5,11a-dihydro-3H-benzo[e]pyrrolo[1,2-a][1,4]diazepine-11acarboxamide (12m). Brown solid. Yield: $280 \mathrm{mg}, 75 \%$. M. p. $144-146{ }^{\circ} \mathrm{C} .{ }^{1} \mathrm{H}$ NMR $(300 \mathrm{MHz}$, $\left.\mathrm{CDCl}_{3}\right)$ ס: 7.99-7.96 (m, 1H), 7.54-7.27 (m, 8H), $6.01(\mathrm{dt}, J=6.3,1.8 \mathrm{~Hz}, 1 \mathrm{H}), 5.85(\mathrm{dt}, J=6.3,1.8$ $\mathrm{Hz}, 1 \mathrm{H}), 5.22(\mathrm{~s}, 1 \mathrm{H}, \mathrm{NH}), 4.69-4.53(\mathrm{~m}, 2 \mathrm{H}), 0.87(\mathrm{~s}, 9 \mathrm{H}) .{ }^{13} \mathrm{C}$ NMR $\{\mathrm{DEPT}-135\}\left(75 \mathrm{MHz}, \mathrm{CDCl}_{3}\right)$

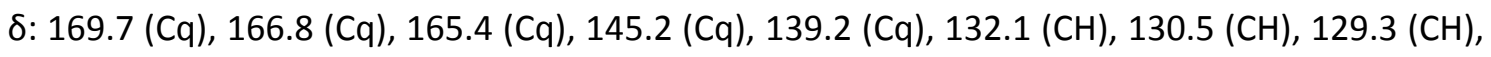
129.2 (CH), $128.1(\mathrm{CH}), 127.8(\mathrm{CH}), 127.3(\mathrm{CH}), 127.0(\mathrm{CH}), 126.3(\mathrm{CH}), 124.9(\mathrm{Cq}), 76.4(\mathrm{Cq})$, $55.2\left(\mathrm{CH}_{2}\right), 51.5(\mathrm{Cq}), 27.9\left(\mathrm{CH}_{3}\right)$. HRMS (ESI-QTOF) $\mathrm{m} / \mathrm{z}$ : calculated for $\mathrm{C}_{23} \mathrm{H}_{24} \mathrm{~N}_{3} \mathrm{O}_{2}\left[\mathrm{M}+\mathrm{H}^{+}\right]$ 374.1863; found 374.1865.

\section{7-Chloro-N-cyclohexyl-5-oxo-11-phenyl-5,11a-dihydro-3H-benzo[e]pyrrolo[1,2-}

a][1,4]diazepine-11a-carboxamide (12n). Brown solid. Yield: $282 \mathrm{mg}, 65 \%$. M. p. 178-180 ${ }^{\circ} \mathrm{C}$.

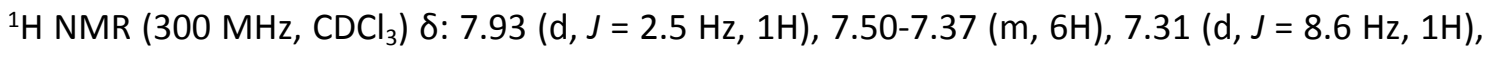
$6.01(\mathrm{dt}, J=6.3,2.1 \mathrm{~Hz}, 1 \mathrm{H}), 5.85(\mathrm{dt}, J=6.3,2.1 \mathrm{~Hz}, 1 \mathrm{H}), 5.56(\mathrm{~d}, J=8.5 \mathrm{~Hz}, 1 \mathrm{H}, \mathrm{NH}), 4.66(\mathrm{dt}, J$ $=17.5,2.2 \mathrm{~Hz}, 1 \mathrm{H}), 4.55(\mathrm{dt}, J=17.5,2.2 \mathrm{~Hz}, 1 \mathrm{H}), 3.41-3.27(\mathrm{~m}, 1 \mathrm{H}), 1.59-0.47(\mathrm{~m}, 10 \mathrm{H}) .{ }^{13} \mathrm{C}$ NMR \{DEPT-135\} (75 MHz, CDCl 3 ) ס: 169.9 (Cq), 166.5 (Cq), 164.1 (Cq), 143.8 (Cq), 138.9 (Cq), 132.2 (CH), $131.9(\mathrm{Cq}), 130.3(\mathrm{CH}), 129.4(\mathrm{CH}), 129.1(\mathrm{CH}), 128.9(\mathrm{CH}), 128.2(\mathrm{CH}), 127.8(\mathrm{CH})$, $127.1(\mathrm{CH}), 126.1(\mathrm{Cq}), 76.2(\mathrm{Cq}), 55.3\left(\mathrm{CH}_{2}\right), 48.6(\mathrm{CH}), 32.3\left(\mathrm{CH}_{2}\right), 32.2\left(\mathrm{CH}_{2}\right), 25.1\left(\mathrm{CH}_{2}\right), 24.8$ $\left(\mathrm{CH}_{2}\right), 24.7\left(\mathrm{CH}_{2}\right)$. HRMS (ESI-QTOF) $\mathrm{m} / \mathrm{z}$ : calculated for $\mathrm{C}_{25} \mathrm{H}_{25} \mathrm{ClN}_{3} \mathrm{O}_{2}\left[\mathrm{M}+\mathrm{H}^{+}\right]$434.1630; found 434.1634. 


\section{8-Chloro-N-cyclohexyl-5-oxo-11-phenyl-5,11a-dihydro-3H-benzo[e]pyrrolo[1,2-}

a][1,4]diazepine-11a-carboxamide (12o). Yellow solid. Yield: $304 \mathrm{mg}, 70 \%$. M. p. $78-80{ }^{\circ} \mathrm{C} .{ }^{1} \mathrm{H}$ $\operatorname{NMR}\left(300 \mathrm{MHz}, \mathrm{CDCl}_{3}\right)$ 8: $7.89(\mathrm{~d}, J=8.5 \mathrm{~Hz}, 1 \mathrm{H}), 7.50-7.37(\mathrm{~m}, 6 \mathrm{H}), 7.24(\mathrm{dd}, J=8.5,2.1 \mathrm{~Hz}$, $1 \mathrm{H}), 6.00(\mathrm{~d}, J=6.3,2.3 \mathrm{~Hz}, 1 \mathrm{H}), 5.84(\mathrm{~d}, J=6.3,2.3 \mathrm{~Hz}, 1 \mathrm{H}), 5.49(\mathrm{~d}, J=8.5 \mathrm{~Hz}, 2 \mathrm{H}, \mathrm{NH}), 4.64$ (dt, $J=17.5,2.2 \mathrm{~Hz}, 1 \mathrm{H}), 4.53(\mathrm{dt}, J=17.5,2.2 \mathrm{~Hz}, 1 \mathrm{H}), 3.39-3.25(\mathrm{~m}, 1 \mathrm{H}), 1.55-0.43(\mathrm{~m}, 10 \mathrm{H})$. ${ }^{13} \mathrm{C}$ NMR $\{$ DEPT-135\} (75 MHz, CDCl $)$ 8: $170.4(\mathrm{Cq}), 166.5$ (Cq), $164.5(\mathrm{Cq}), 146.1(\mathrm{Cq}), 138.8$ (Cq), $138.0(\mathrm{Cq}), 131.0(\mathrm{CH}), 130.2(\mathrm{CH}), 129.5(\mathrm{CH}), 128.2(\mathrm{CH}), 127.8(\mathrm{CH}), 127.2(\mathrm{CH}), 127.0$ $(\mathrm{CH}), 126.5(\mathrm{CH}), 123.2(\mathrm{Cq}), 76.3(\mathrm{Cq}), 55.3\left(\mathrm{CH}_{2}\right), 48.6(\mathrm{CH}), 32.3\left(\mathrm{CH}_{2}\right), 25.1\left(\mathrm{CH}_{2}\right), 24.8\left(\mathrm{CH}_{2}\right)$, $24.6\left(\mathrm{CH}_{2}\right)$. HRMS (ESI-QTOF) $\mathrm{m} / \mathrm{z}$ : calculated for $\mathrm{C}_{25} \mathrm{H}_{25} \mathrm{ClN}_{3} \mathrm{O}_{2}\left[\mathrm{M}+\mathrm{H}^{+}\right]$434.1630; found 434.1634.

\section{$N$-Cyclohexyl-1-methylene-5-oxo-11-phenyl-2,3,5,11a-tetrahydro-1H-benzo[e]pyrrolo[1,2-} a][1,4]diazepine-11a-carboxamide (12p). Brown solid. Yield: 248 mg, 60\%. M. p. 154-156 ${ }^{\circ} \mathrm{C}$. ${ }^{1} \mathrm{H}$ NMR $\left(300 \mathrm{MHz}, \mathrm{CDCl}_{3}\right) \delta: 7.96(\mathrm{~d}, J=7.9 \mathrm{~Hz}, 1 \mathrm{H}), 7.54-7.26(\mathrm{~m}, 8 \mathrm{H}), 5.49(\mathrm{~d}, J=8.2 \mathrm{~Hz}, 1 \mathrm{H}$, $\mathrm{NH}), 5.32(\mathrm{~s}, 1 \mathrm{H}), 5.17(\mathrm{~s}, 1 \mathrm{H}), 4.15(\mathrm{t}, J=10.7 \mathrm{~Hz}, 1 \mathrm{H}), 3.81-3.71(\mathrm{~m}, 1 \mathrm{H}), 3.32-3.22(\mathrm{~m}, 1 \mathrm{H})$, $2.51(\mathrm{dd}, J=15.6,7.3 \mathrm{~Hz}, 1 \mathrm{H}), 2.32-2.20(\mathrm{~m}, 1 \mathrm{H}), 1.55-0.50(\mathrm{~m}, 10 \mathrm{H}) .{ }^{13} \mathrm{C}$ NMR $\{$ DEPT-135\} $(75$ $\left.\mathrm{MHz}, \mathrm{CDCl}_{3}\right)$ ס: $169.4(\mathrm{Cq}), 166.3(\mathrm{Cq}), 166.2(\mathrm{Cq}), 146.5(\mathrm{Cq}), 145.3(\mathrm{Cq}), 137.8(\mathrm{Cq}), 132.3$ $\left(\mathrm{CH}_{\mathrm{Ar}}\right), 129.7\left(\mathrm{CH}_{\mathrm{Ar}}\right), 129.2\left(\mathrm{CH}_{\mathrm{Ar}}\right), 128.4\left(\mathrm{CH}_{\mathrm{Ar}}\right), 127.6\left(\mathrm{CH}_{\mathrm{Ar}}\right), 127.1\left(\mathrm{CH}_{\mathrm{Ar}}\right), 126.3\left(\mathrm{CH}_{\mathrm{Ar}}\right), 125.1$ (Cq), $114.8\left(\mathrm{CH}_{2}\right), 73.1(\mathrm{Cq}), 48.5(\mathrm{CH}), 46.9\left(\mathrm{CH}_{2}\right), 32.2\left(\mathrm{CH}_{2}\right), 32.1\left(\mathrm{CH}_{2}\right), 30.6\left(\mathrm{CH}_{2}\right), 25.2\left(\mathrm{CH}_{2}\right)$, $24.8\left(\mathrm{CH}_{2}\right), 24.7\left(\mathrm{CH}_{2}\right)$. HRMS (ESI-QTOF) $m / z$ : calculated for $\mathrm{C}_{26} \mathrm{H}_{28} \mathrm{~N}_{3} \mathrm{O}_{2}\left[\mathrm{M}+\mathrm{H}^{+}\right]$414.2176; found 414.2182 .

\section{$N$-(tert-Butyl)-1-methylene-5-oxo-11-phenyl-2,3,5,11a-tetrahydro-1H-benzo[e]pyrrolo[1,2-}

a][1,4]diazepine-11a-carboxamide (12q). White solid. Yield: $225 \mathrm{mg}, 58 \%$. M. p. $102-104{ }^{\circ} \mathrm{C} .{ }^{1} \mathrm{H}$ NMR $\left(300 \mathrm{MHz}, \mathrm{CDCl}_{3}\right) \delta: 7.96(\mathrm{dd}, J=7.9,1.7 \mathrm{~Hz}, 1 \mathrm{H}), 7.52-7.20(\mathrm{~m}, 8 \mathrm{H}), 5.34(\mathrm{~d}, J=1.7 \mathrm{~Hz}$, $1 \mathrm{H}, \mathrm{NH}), 5.16-5.14(\mathrm{~m}, 2 \mathrm{H}), 4.13(\mathrm{ddd}, J=11.9,10.0,2.5 \mathrm{~Hz}, 1 \mathrm{H}), 3.65$ (ddd, $J=11.9,10.0,7.6$ $\mathrm{Hz}, 1 \mathrm{H}), 2.53-2.45(\mathrm{~m}, 1 \mathrm{H}), 2.30-2.17(\mathrm{~m}, 1 \mathrm{H}), 0.87(\mathrm{~s}, 9 \mathrm{H}) .{ }^{13} \mathrm{C}$ NMR $\{\mathrm{DEPT}-135\}\left(75 \mathrm{MHz}, \mathrm{CDCl}_{3}\right)$

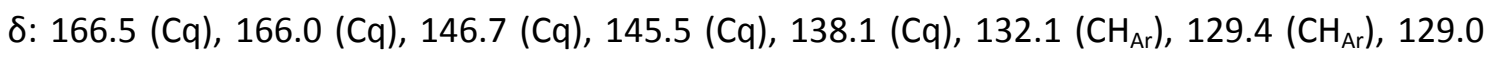


$\left(\mathrm{CH}_{\mathrm{Ar}}\right), 128.3\left(\mathrm{CH}_{\mathrm{Ar}}\right), 127.5\left(\mathrm{CH}_{\mathrm{Ar}}\right), 127.2\left(\mathrm{CH}_{\mathrm{Ar}}\right), 126.2\left(\mathrm{CH}_{\mathrm{Ar}}\right), 125.5(\mathrm{Cq}), 114.1\left(\mathrm{CH}_{2}\right), 73.3(\mathrm{Cq})$, $51.2(\mathrm{Cq}), 46.4\left(\mathrm{CH}_{2}\right), 30.6\left(\mathrm{CH}_{2}\right), 27.7\left(\mathrm{CH}_{3}\right)$. HRMS (ESI-QTOF) $\mathrm{m} / \mathrm{z}$ : calculated for $\mathrm{C}_{24} \mathrm{H}_{26} \mathrm{~N}_{3} \mathrm{O}_{2}$ $\left[\mathrm{M}+\mathrm{H}^{+}\right]$388.2020; found 388.2031.

\section{8-Chloro- $\mathrm{N}$-cyclohexyl-1-methylene-5-oxo-11-phenyl-2,3,5,11a-tetrahydro-1H-} benzo[e]pyrrolo[1,2-a][1,4]diazepine-11a-carboxamide (12r). Brown solid. Yield: $278 \mathrm{mg}$, $62 \%$. M. p. $118-120{ }^{\circ} \mathrm{C} .{ }^{1} \mathrm{H}$ NMR $\left(300 \mathrm{MHz}, \mathrm{CDCl}_{3}\right) \delta: 7.90(\mathrm{~d}, J=8.5 \mathrm{~Hz}, 1 \mathrm{H}), 7.46-7.23(\mathrm{~m}, 7 \mathrm{H})$, $5.37(\mathrm{~d}, J=8.4 \mathrm{~Hz}, 1 \mathrm{H}, \mathrm{NH}), 5.31(\mathrm{~d}, J=2.3 \mathrm{~Hz}, 1 \mathrm{H}), 5.19(\mathrm{~d}, J=2.3 \mathrm{~Hz}, 1 \mathrm{H}), 4.11$ (ddd, $J=11.9$, 9.3, $2.5 \mathrm{~Hz}, 1 \mathrm{H}$ ), $3.72(\mathrm{ddd}, J=11.9,9.3,7.7 \mathrm{~Hz}, 1 \mathrm{H}), 3.40-3.27(\mathrm{~m}, 1 \mathrm{H}), 2.52$ (ddd, $J=15.5,7.7$, $2.5 \mathrm{~Hz}, 1 \mathrm{H}), 2.32-2.19(\mathrm{~m}, 1 \mathrm{H}), 1.67-0.53(\mathrm{~m}, 10 \mathrm{H}) .{ }^{13} \mathrm{C}$ NMR $\left\{\right.$ DEPT-135\} $\left(75 \mathrm{MHz}, \mathrm{CDCl}_{3}\right) \delta$ : $170.2(\mathrm{Cq}), 166.2(\mathrm{Cq}), 165.3(\mathrm{Cq}), 146.4(\mathrm{Cq}), 138.1(\mathrm{Cq}), 137.7(\mathrm{Cq}), 131.1(\mathrm{CH}), 129.3(\mathrm{CH})$, $128.3(\mathrm{CH}), 127.6(\mathrm{CH}), 127.0(\mathrm{CH}), 126.4(\mathrm{CH}), 123.7(\mathrm{Cq}), 114.6\left(\mathrm{CH}_{2}\right), 73.2(\mathrm{Cq}), 48.5(\mathrm{CH})$, 46.8 $\left(\mathrm{CH}_{2}\right), 32.3\left(\mathrm{CH}_{2}\right), 32.2\left(\mathrm{CH}_{2}\right), 30.6\left(\mathrm{CH}_{2}\right), 25.2\left(\mathrm{CH}_{2}\right), 24.8\left(\mathrm{CH}_{2}\right), 24.7\left(\mathrm{CH}_{2}\right) . \mathrm{HRMS}(\mathrm{EI}) \mathrm{m} / \mathrm{z}$ : calculated for $\mathrm{C}_{26} \mathrm{H}_{27} \mathrm{ClN}_{3} \mathrm{O}_{2}\left[\mathrm{M}+\mathrm{H}^{+}\right]$448.1786; found 448.1793 .

\section{Associated content}

The Supporting Information is available free of charge on the ACS Publications website at DOI: $x x . x x x x / a c s . j o c . x x x x x x x$.

Copies of ${ }^{1} \mathrm{H},{ }^{13} \mathrm{C}$ and DEPT-135 NMR spectra and high-resolution mass spectra (PDF).

X-ray chrystallographic data for compound 12i (CIF). CCDC 1975921.

\section{Author information}

\section{Corresponding author}

*E-mail: magaval@ubu.es

\section{ORCID}

María García-Valverde: 0000-0002-3990-8388. 
Roberto Quesada: 0000-0003-2764-7157

Israel Carreira Barral: 0000-0002-4835-8752

Notes

The authors declare no competing financial interest.

\section{Acknowledgments}

Funding from Consejería de Educación de la Junta de Castilla y León (project BU075G19) is gratefully acknowledged.

\section{References}

(1) (a) Gerratana, B. Biosynthesis, synthesis, and biological activities of pyrrolobenzodiazepines. Med. Res. Rev. 2012, 32, 254-293. (b) Cipolla, L.; Araújo, A. C.; Airoldi, C.; Bini, D. Pyrrolo[2,1-c][1,4]benzodiazepine as a scaffold for the design and synthesis of antitumour drugs. Anti-Cancer Agents Med. Chem. 2009, 9, 1-31.

(2) (a) Kohn, K. W.; Glaubiger, D.; Spears, C. L. The reaction of anthramycin with DNA: II. Studies of kinetics and mechanism, Biochim. Biophys. Acta 1974, 361, 288-302. (b) Hurley, L. H.; Gairola, C.; Zmijewski, M. Pyrrolo(1,4)benzodiazepine antitumor antibiotics. In vitro interaction of anthramycin, sibiromycin and tomaymycin with DNA using specifically radiolabelled molecules, Biochim. Biophys. Acta 1977, 475, 521-535.

(3) Nekkanti, S.; Tokala, R.; Shankaraiah, N. Targeting DNA minor groove by hybrid molecules as anticancer agents, Curr. Med. Chem. 2017, 24, 2887-2907.

(4) Mantaj, J.; Jackson, P. J. M.; Rahman, K. M.; Thurston, D. E. From anthramycin to pyrrolobenzodiazepine (PBD)-containing antibody-drug conjugates (ADCs). Angew. Chem. Int. Ed. 2017, 56, 462-488. 
(5) Leimgruber, W.; Stefanovic, V.; Schenker, F.; Karr, A.; Berger, J. Isolation and characterization of anthramycin, a new antitumour antibiotic. J. Am. Chem. Soc. 1965, 87, 5791-5793.

(6) Konishi, M.; Hatori, M.; Tomita, K.; Sugawara, M.; Ikeda, C.; Nishiyama, Y.; Imanishi, H.; Miyaki, T.; Kawaguchi, H. Chicamycin, a new antitumor antibiotic. J. Antibiot. 1984, 37, 191199.

(7) Arima, K.; Kohsaka, M.; Tamura, G.; Imanaka, H.; Sakai, H. Studies on tomaymycin, a new antibiotic. I. Isolation and properties of tomaymycin, J. Antibiot. 1972, 25, 437-444.

(8) Gregson, S. J.; Howard, P. W.; Corcoran, K. E.; Barcella, S.; Yasin, M. M.; Hurst, A. A.; Jenkins, T. C.; Kelland, L. R.; Thurston, D. E. Effect of C2-exo unsaturation on the cytotoxicity and DNA-binding reactivity of pyrrolo[2,1-c][1,4]benzodiazepines. Bioorg. Med. Chem. Lett. $2000,10,1845-1847$

(9) Gregson, S. J.; Howard, P. W.; Barcella, S.; Nakamya, A.; Jenkins, T. C.; Kelland, L. R.; Thurston, D. E. Effect of C2/C3-endo unsaturation on the cytotoxicity and DNA-binding reactivity of pyrrolo[2,1-c][1,4]benzodiazepines. Bioorg. Med. Chem. Lett. 2000, 10, 1849-1851.

(10) (a) Thurston, D. E.; Bose, D. S. Synthesis of DNA-interactive pyrrolo[2,1c][1,4]benzodiazepines. Chem. Rev. 1994, 94, 433-465. (b) Antonow, D.; Thurston, D. E. Synthesis of DNA-interactive pyrrolo[2,1-c][1,4]benzodiazepines (PBDs). Chem. Rev. 2011, 111, 2815-2864.

(11) Hartley, J. A. The development of pyrrolobenzodiazepines as antitumour agents. Expert Opin. Invest. Drugs 2011, 20, 733-744.

(12) Hu, W.-P.; Wang, J.-J.; Lin, F.-L.; Lin, Y.-C.; Lin, S.-R.; Hsu, M.-H. An efficient synthesis of pyrrolo[2,1-c][1,4]benzodiazepine. Synthesis of the antibiotic DC-81. J. Org. Chem. 2001, 66, 2881-2883. 
(13) Eguchi, S.; Yamashita, K.; Matsushita, Y.; Kakehi, A. Facile synthesis of 1,4-benzodiazepin5-one derivatives via intramolecular aza-Wittig reaction. Application to an efficient synthesis of O-benzyl DC-81. J. Org. Chem. 1995, 60, 4006-4012.

(14) Miyamoto, M.; Kondo, S.; Naganawa, H.; Maeda, K.; Ohno, M.; Umezawa, H. Structure and synthesis of neothramycin. J. Antibiot. 1977, 30, 340-343.

(15) Fukuyama, T.; Lin, S.-C.; Li, L. Facile reduction of ethyl thiol esters to aldehydes: application to a total synthesis of (+)-neothramycin A methyl ether. J. Am. Chem. Soc. 1990, $112,7050-7051$.

(16) Fukuyama, T.; Liu, G.; Linton, S. D.; Lin, S.-C.; Nishino, H. Total synthesis of (+)porothramycin B. Tetrahedron Lett. 1993, 34, 2577-2580.

(17) (a) Pertejo, P.; García-Valverde, M.; Peña, P.; Cordero, N. A.; Torroba, T.; González-Ortega, A. Experimental and theoretical studies on the effect of the oxo group in 1,4-benzodiazepines. Org. Biomol. Chem. 2014, 12, 4905-4916. (b) Pertejo, P.; Peña-Calleja, P.; Carreira-Barral, I.; Quesada, R.; Cordero, N. A.; Rodríguez, F. J.; García-Valverde, M. Novel pyrrolobenzodiazepine and pyrroloquinazoline scaffolds synthesized by a simple and highly selective Ugi/cyclization sequence. Org. Biomol. Chem. 2017, 15, 7549-7557.

(18) Dénès, F.; Pérez-Luna, A.; Chemla, F. Addition of metal enolate derivatives to unactivated carbon-carbon multiple bonds. Chem. Rev. 2010, 110, 2366-2447.

(19) Zimmer, R.; Reissig, H.-U. Modern Allene Chemistry. Krause, N. and Hashmi, A. S., Eds., Wiley-VCH, 2004, ch. 8, pp. 425-492.

(20) (a) Pérez-Sestelo, J.; Sarandeses, L. A.; Martínez, M. M.; Alonso-Marañón, L. Indium(III) as $\pi$-acid catalyst for the electrophilic activation of carbon-carbon unsaturated systems. Org. Biomol. Chem. 2018, 16, 5733-5747. (b) Pathipati, S. R.; van der Werf, A.; Selander, N. Indium(III)-catalyzed transformations of alkynes: recent advances in carbo- and heterocyclization reactions. Synthesis, 2017, 49, 4931-4941. 
(21) Alabugin, I. V.; Gilmore, K.; Manoharan, M. Rules for anionic and radical ring closure of alkynes. J. Am. Chem. Soc. 2011, 133, 12608-12623.

(22) Polindara-García, L. A.; Miranda, L. D. Two-step synthesis of 2,3-dihydropyrroles via a formal 5-endo cycloisomerization of Ugi 4-CR/propargyl adducts. Org. Lett. 2012, 14, 54085411.

(23) Polindara-García, L. A.; Vázquez, A. Combinatorial synthesis of nicotine analogs using an Ugi 4-CR/cyclization-reduction strategy. Org. Biomol. Chem. 2014, 12, 7068-7082.

(24) Lu, T.; Lu, Z.; Ma, Z.X.; Zhang, Y.; Hsung, R. P. Allenamides: A Powerful and Versatile Building Block in Organic Synthesis. Chem. Rev. 2013, 113, 4862-4904.

(25) García, L.; Sendra, J.; Miralles, N.; Reyes, E.; Carbó, J. J.; Vicario, J. L.; Fernández, E. Transition-metal-free stereoselective borylation of allenamides. Chem. Eur. J. 2018, 24, 1405914063.

(26) Wang, Y.; Cai, P. -J.; Yum Z. -X. Carbanion translocations via intramolecular proton transfers: A quantum chemical study. J. Org. Chem. 2017, 82, 4604-4612.

(27) Boggs, J. E.; Kim, M. G. The structures of 1-, 2- and 3-pyrroline. J. Mol. Struct. 1985, 119, 271-279.

(28) (a) Vandavasi, J. K.; Hu, W.-P.; Senadi, G. C.; Boominathan, S. S. K.; Chen, H.-Y.; Wang, J.-J. $\mathrm{A} \mathrm{K}_{2} \mathrm{CO}_{3}$-mediated regioselective synthesis of indole/pyrrole-fused 1,4-oxazines: an unexpected indole-fused azlactone synthesis. Eur. J. Org. Chem. 2014, 6219-6226. (b) Pandey, S.; Kumar, S. V.; Kant, R.; Chauhan, P. M. S. Base mediated 7-exo-dig intramolecular cyclization of Ugipropargyl precursors: a highly efficient and regioselective synthetic approach toward diverse 1,4-benzoxazepine-5(2H)-ones. Org. Biomol. Chem. 2014, 12, 5346-5350. 
(29) Flores-Constante, G.; Sánchez-Chávez, A. C.; Polindara-García, L. A. A convenient synthesis of 1,2-disubstituted-cis-3,4-dihydroxypyrrolidines via an Ugi-four-componentreaction/cycloisomerization/dihydroxylation protocol. Eur. J. Org. Chem. 2018, 4586-4591.

(30) Pertejo, P.; Corres, N.; Torroba, T.; García-Valverde, M. Reversal of diastereoselectivity in the synthesis of peptidomimetic 3-carboxamide-1,4-benzodiazepin-5-ones. Org. Lett. 2015, 17, 612-615. 